\title{
Memory effect in anti-de Sitter spacetime
}

\author{
Chong-Sun Chu $\odot^{*}$ and Yoji Koyama ${ }^{\dagger}$ \\ Physics Division, National Center for Theoretical Sciences, National Tsing-Hua University, \\ Hsinchu, 30013, Taiwan \\ and Department of Physics, National Tsing-Hua University, Hsinchu 30013, Taiwan
}

(Received 29 September 2019; published 18 November 2019)

\begin{abstract}
The geodesic deviation of a pair of test particles is a natural observable for the gravitational memory effect. Nevertheless in curved spacetime, this observable is plagued with various issues that need to be clarified before one can extract the essential part that is related to the gravitational radiation. In this paper we consider the anti-de Sitter (AdS) space as an example and analyze this observable carefully. We show that by employing the Fermi normal coordinates around the geodesic of one of the particles (i.e., the standard free falling frame attached to this particle), one can elegantly separate out the curvature contribution of the background spacetime to the geodesic deviation from the contribution of the gravitational wave. The gravitational wave memory obtained this way depends linearly and locally on the retarded metric perturbation caused by the gravitational wave, and, remarkably, it takes on exactly the same formula as in the flat case. To determine the memory, in addition to the standard tail contribution to the gravitational radiation, one needs to take into account the contribution from the reflected gravitational wave off the AdS boundary. For general curved spacetime, our analysis suggests that the use of a certain coordinate system adapted to the local geodesic (e.g., the Fermi normal coordinates system in the AdS case) would allow one to dissect the geodesic deviation of test particles and extract the relevant contribution to define the memory due to gravitational radiation.
\end{abstract}

DOI: 10.1103/PhysRevD.100.104034

\section{INTRODUCTION}

Passage of a gravitational wave $(\mathrm{GW})$ can induce a permanent displacement in the relative separation of a pair of test particles which serves as a gravitational wave detector. This phenomenon is known as the gravitational memory effect [1]. In a flat background, the net relative displacement $\Delta D_{\mu}$ between the test particles, after passage of the gravitational radiation, is given by

$$
\Delta D_{\mu}=\frac{1}{2} \Delta h_{\mu \nu}^{T T} D^{\nu}
$$

where $D^{\nu}$ is the initial separation of the pair of test particles, and $\Delta h_{\mu \nu}^{T T}$ is the net change in the metric perturbations in the transverse-traceless gauge. In flat spacetime, the memory formula (1) is simple. The gravitational memory $\Delta D_{\mu}$ is determined entirely in terms of the metric

*cschu@phys.nthu.edu.tw

koyama811@gmail.com

Published by the American Physical Society under the terms of the Creative Commons Attribution 4.0 International license. Further distribution of this work must maintain attribution to the author(s) and the published article's title, journal citation, and DOI. Funded by SCOAP ${ }^{3}$. perturbation, which, in linearized gravity, can be solved in terms of the retarded Green function $G_{R}$. Schematically, without going into details about gauge fixing and decomposition into irreducible components, the retarded Green function $G_{R}$ satisfies

$$
\square_{x} G_{R}\left(x, x^{\prime}\right)=-\delta^{(4)}\left(x, x^{\prime}\right),
$$

where $\square$ is a second order differential operator in which the linearized Einstein equation can be written as $\square_{x} h_{\mu \nu}=-16 \pi G T_{\mu \nu}$. As a result, we have

$$
h_{\mu \nu}(x)=16 \pi G \int d^{4} x^{\prime} G_{R}\left(x, x^{\prime}\right) T_{\mu \nu}\left(x^{\prime}\right)
$$

and the study of the properties of the memory effect can be phrased entirely in terms of the properties of the retarded Green function. For gravitational wave generated by a source in a localized region of spacetime, $\Delta h_{\mu \nu}^{T T}$ is of Coulomb type [2], i.e., $\Delta h_{\mu \nu}^{T T} \sim 1 / r$ at large distance $r$ of the detector from the source. For example, the collision or explosion of a collection of freely moving particles produces the perturbation $[2,3]$ 


$$
\Delta h_{\mu \nu}^{T T}=\frac{1}{r} \Delta\left[\sum_{A=1}^{N} \frac{4 M_{A}}{\sqrt{1-v_{A}^{2}}}\left(\frac{v_{A \mu} v_{A \nu}}{1-v_{A} \cos \theta_{A}}\right)^{T T}\right],
$$

where $M_{A}$ and $v_{A}$ are the mass and the velocity of each freely moving massive body, respectively, and $\theta_{A}$ is the angle between the source and the detector. $T T$ denotes the transverse-traceless part of the expression. Memory effect in flat space and its properties as produced by various kinds of massive and massless particle sources were recently discussed in [4-8]. Detectability of the memory effect from gravitational wave signals associated with binary black hole mergers was discussed in $[3,9]$. Recent discussions about the detectability with LISA, pulsar timing arrays or LIGO can be found in [10-13].

We are interested in the memory effect in curved spacetime. There are various motivations for this interest. First, our Universe is curved and not flat. It was de Sitterlike at the time of inflation. Currently it is described by a Friedmann-Lemaître-Robertson-Walker (FLRW) spacetime. One can imagine that the observation of gravitational memory effect may provide valuable information on the structure of the Universe at various stages of its development. Theoretically, gravitational memory effect in flat space is related to the asymptotic Bondi-Metzner-Sachs (BMS) symmetry [14,15] and the infrared properties of gravity $[16,17]$. It is interesting to understand how much of this story may carry through in a curved background spacetime.

While the memory effect in de Sitter (dS) and FLRW spacetimes has been extensively studied, memory effect in anti-de Sitter (AdS) space has been considered much less in the literature (see, e.g., [18-25]; also [26] for a study of the memory effect in gauge theory). Although the memory effect in AdS space is currently less motivated from an observational point of view than that in dS and FLRW spacetimes, theoretically it is an interesting subject. First, as we will demonstrate in this paper, the study of memory effect in the AdS spacetime gives us useful insights on better ways to think about and analyze the memory effect in general curved spacetime. Besides, the existence of boundary in AdS also offers an interesting opportunity to study how the reflected gravitational wave may affect the gravitational memory. Moreover, the study of AdS memory is potentially related to the other gravitational phenomena such as asymptotic symmetries of spacetime and AdS/CFT correspondence [27]. These are some of the reasons behind that form the main motivations of this work.

The study of gravitational memory in curved spacetime is however much more complicated and a number of effects not occurring in the flat spacetime needed to be taken into account.

(1) In curved spacetime, the simple result (1) no longer holds and the net change $\Delta D_{\mu}$ in the geodesic separation of test particles has to be obtained from solving the geodesic derivation equation. Due to the presence of nontrivial spacetime curvature, $\Delta D_{\mu}$ generally involves integration over the history of the motion of the particles and takes a much more complicated nonlocal form compared to (1). In spacetime without boundary, one can gain huge simplification by making the observation at large distance and at large time so that a local unintegrated expression for $\Delta D_{\mu}$ is obtained. ${ }^{1}$ This however does not work for the AdS space due to the presence of boundary, and it seems that the expression of the memory in AdS space will necessarily be much more complicated.

(2) In a curved spacetime, the geodesic separation between the pair of particles gets contributions from both the background curvature (backscattering by the gravitational potential created by the background curvature) as well as the gravitational wave. Therefore in order to have a proper definition of memory due to gravitational wave, it is important to separate the contribution of the background curvature from the contribution of the gravitational wave. In this work, we show that the above two issues can be resolved elegantly by adopting a particular choice of coordinate system to make the observation of memory.

(3) Another subtlety in curved spacetime is the presence of the tail term in the retarded Green function, i.e., propagation of gravitational waves less than the speed of light, besides the propagation of gravitational waves at the speed of light which we will call the direct term. Let us comment on these contributions to the memory in various spacetime such as the dS spacetime [18,20], decelerating FLRW spacetime with future null infinity [21], spatially flat FLRW cosmologies [22], and the $\Lambda \mathrm{CDM}$ cosmologies [23] that have been considered in the literature. For the spatially flat FLRW spacetime [22] with localized source, the authors proposed to continue to use the flat space criteria of the presence of a derivative of a delta function in the Riemann curvature as a way to characterize the gravitational memory for the spatially flat FLRW spacetime. By construction, the tail contribution was excluded in this characterization of the memory. For the decelerating FLRW spacetime [21], it was found that the tail term in the retarded potential is subleading in the $1 / r$ expansion at the future null infinity and the memory effect is given entirely by the direct term [21]. In these two cases, the direct term contribution to the memory effect is simple and can be written in the same form as the flat case up to an multiplicative factor of an inverse power of the scale factor $a(\tau)$ at the time of detection

\footnotetext{
${ }^{1}$ See e.g., [19] for the dS case to see how the nonlocal expression [Eq. (5) there] can be reduced to a local expression [Eq. (6)] there at large time.
} 
when the source and the detector are placed at the same proper distance as that in flat space at the time of gravitational wave emission. On the other hand, for the de Sitter space, it was found that for even dimensions higher than $2[19,24,25]$, the gravitational wave tail contributes significantly to the memory effect. ${ }^{2}$ Note that in this case the retarded potential is expanded in terms of the conformal time $\eta$, instead of $1 / r$, in order to approach the future infinity [25]. At $\eta=0$, the tail and direct contributions are equal in size but opposite in sign and thus they cancel out each other, leaving the higher order terms in $H$, in the future infinity. From these results, one can learn the lesson that both the direct term and the tail term depend rather sensitively on the asymptotic geometry of the spacetime where the observation of the memory is made. In this regard, AdS spacetime has a boundary and its asymptotics is completely different. The determination of both the direct and the tail contributions at generic finite location in AdS space is another motivation of this work.

(4) AdS space is also special since it has a boundary and reflection may occur, and the reflected wave may affect the observed memory effect in a significant way. We will show in this paper that, depending on the location of the detector, the reflected gravitational wave may make a significant contribution to the observed gravitational memory.

In this work, we study memory effect on fourdimensional AdS space in the Poincaré coordinates. We restrict ourselves to the linear order of metric perturbation around the vacuum AdS space. As the background spacetime is nontrivial, the geodesic separation $D^{\mu}$ is a gauge dependent quantity and it is necessary to specify an observer which made the result of memory as transparent physically as possible. We find that by employing the Fermi normal coordinates around the geodesic of one of the particles, one can elegantly separate out the curvature contribution of the background spacetime from those of the gravitational wave. Remarkably, the obtained gravitational wave memory takes on exactly the same formula (1) as in the flat case and has a simple local and factorized dependence on the retarded metric perturbation caused by the gravitational wave. This simple formula allows us to determine the memory entirely in terms of the waveform of the retarded gravitational radiation. As we mentioned above, in AdS space the retarded propagator of metric perturbation contains a tail term. Aside from the tail term, AdS space has a distinctive feature that the gravitational waves reach the infinity (AdS boundary) in a finite time and then get reflected back to the bulk spacetime. As a consequence, in addition to the original gravitational wave,

\footnotetext{
${ }^{2}$ It is known that the tail term vanishes for odd dimensional de Sitter spacetime.
}

there will also be a gravitational wave reflected at the AdS boundary, each accompanied by its respective tail term. The net memory is given by the sum of all these contributions.

For general curved spacetime, our analysis suggests that the use of a certain coordinate system that is adapted to the local geodesic (e.g., the Fermi normal coordinates system in the AdS case) would allow one to dissect the geodesic deviation of test particles and extract the relevant contribution to define the memory effect due to gravitational radiation. This is an interesting direction to further explore [28].

The organization of this article is as follows. As advertised, the retarded metric perturbation plays an important role in determining the memory in AdS space, therefore we will first start in Sec. II with the construction of the retarded solution for the linear metric perturbation in AdS spacetime. Following Wald and Tolish [7], we will consider the localized energy source as an example and work out the retarded wave solution. In Sec. III A, we consider the use of the Fermi normal coordinates and find that the background curvature contribution to the geodesic deviation can be easily disentangled and subtracted away. The remaining part of the geodesic deviation (67) depends on the retarded gravitational wave linearly and locally, and in fact takes on exactly the same form (1) as in the flat case. This is true for any finite time. This is quite remarkable and is one of the main results of this work. In Sec. III B, we take into account the tail term and the reflected wave and analyze the memory effect. As the reflected wave plays an important role, the effect of memory depends crucially on whether the detector is receiving the reflected wave or not. We show that for an observer that receive the reflected wave, the memory effect got canceled out completely. Hence it is interesting that memory effect is different for different locations of the gravitational wave detector in the AdS spacetime. In Sec. III C, we consider the asymptotic form of the memory near the AdS boundary and find that it has a delta function singularity localized on the light cone from the source. In Secs. III D and III E, we construct the AdS shock wave by taking a certain limit of our perturbed metric. We find that the velocity memory for the AdS shock wave picks up a kink contribution $u \theta(u)$ in addition to the jump $\theta(u)$ and pulse term $\delta(u)$ which are present in the flat case. Section IV contains our conclusion and some discussions. Some of the more technical details of the analysis are contained in the Appendixes.

\section{LINEAR PERTURBATION IN AdS SPACE}

Consider an $n$-dimensional AdS space. In the Poincaré coordinates, the line element is given by

$d s^{2}=\bar{g}_{\mu \nu} d x^{\mu} d x^{\nu}=\frac{L^{2}}{y^{2}}\left(-d t^{2}+\left(d x^{i}\right)^{2}+d y^{2}\right)$,

$\bar{g}_{\mu \nu}=\frac{L^{2}}{y^{2}} \eta_{\mu \nu}$ 
with $-\infty<t, x^{i}<\infty, 0<y<\infty$. Here the subscripts $i, j=1, \ldots, n-2$ and $\mu, \nu=0, \ldots, n-2, y$, and $L$ is the AdS radius. The AdS boundary is located at $y=0$ and the AdS horizon is at $y=\infty$. Below we will use the notation for indices such that $a, b=0, \ldots, n-2$ and $r, s=1, \ldots$, $n-2, y$.

\section{A. Retarded solution}

We consider perturbation $\gamma_{\mu \nu}$ around the background AdS metric,

$$
g_{\mu \nu}=\bar{g}_{\mu \nu}+\gamma_{\mu \nu} .
$$

It is convenient to introduce the perturbation $\psi_{\mu \nu}$ from $\gamma_{\mu \nu}$ as defined by

$$
\psi_{\mu \nu}=\gamma_{\mu \nu}-\frac{1}{2} \bar{g}_{\mu \nu} \gamma, \quad \gamma:=\bar{g}^{\mu \nu} \gamma_{\mu \nu} .
$$

We impose the following gauge conditions analogous to the one adopted in de-Sitter space [29]

$$
\bar{\nabla}_{\nu} \psi_{\mu}^{\nu}=-\frac{2}{y} \psi_{\mu}^{y},
$$

where $\bar{\nabla}_{\mu}$ is the covariant derivative with respect to $\bar{g}_{\mu \nu}$. In terms of $\psi^{\nu}{ }_{\mu}$, the linearized Einstein equation with a source is

$$
\begin{gathered}
\partial^{2} \psi^{\nu}{ }_{\mu}-\frac{n-2}{y} \partial_{y} \psi^{\nu}{ }_{\mu}+\frac{1}{y^{2}}\left((n-2)\left(\delta^{\nu}{ }_{y} \psi^{y}{ }_{\mu}+\delta^{y}{ }_{\mu} \psi^{\nu}{ }_{y}\right)\right. \\
\left.-2 \delta^{\nu}{ }_{y} \delta^{y}{ }_{\mu} \psi\right)=-\frac{16 \pi G L^{2}}{y^{2}} T^{\nu}{ }_{\mu},
\end{gathered}
$$

where $\psi:=\bar{g}^{\mu \nu} \psi_{\mu \nu}, \partial^{2}=\eta^{\rho \sigma} \partial_{\rho} \partial_{\sigma}, \quad G$ is the Newton constant and $T^{\nu}{ }_{\mu}$ stands for perturbative matter energymomentum tensor.

It is convenient to introduce a rescaled perturbation where its indices are raised and lowered by $\eta_{\mu \nu}$,

$$
\chi_{\mu \nu}:=\frac{y^{2}}{L^{2}} \bar{g}_{\mu \rho} \psi_{\nu}^{\rho}{ }_{\nu}=\eta_{\mu \rho} \psi_{\nu}^{\rho}, \quad \chi:=\eta^{\mu \nu} \chi_{\mu \nu},
$$

and in which the original perturbation $\gamma_{\mu \nu}$ can be written as

$$
\gamma_{\mu \nu}=\frac{L^{2}}{y^{2}}\left(\chi_{\mu \nu}-\frac{1}{2} \eta_{\mu \nu} \chi_{\rho}^{\rho}\right) .
$$

$\chi_{\mu \nu}$ satisfies the linearized Einstein equation,

$$
\begin{gathered}
\partial^{2} \chi_{\mu \nu}-\frac{n-2}{y} \partial_{y} \chi_{\mu \nu}+\frac{1}{y^{2}}\left((n-2)\left(\eta_{y \nu} \chi_{\mu y}+\eta_{\mu \nu} \chi_{y \nu}\right)\right. \\
\left.-2 \eta_{y \nu} \eta_{\mu \nu} \chi\right)=-\frac{16 \pi G L^{2}}{y^{2}} T^{\nu}{ }_{\mu} .
\end{gathered}
$$

Defining $\tilde{\chi}=\chi_{\rho}^{\rho}-(n-2) \chi_{y y}$, Eq. (12) can be decomposed into three independent equations:

$$
\begin{gathered}
\partial^{2} \chi_{a b}-\frac{n-2}{y} \partial_{y} \chi_{a b}=-\frac{16 \pi G L^{2}}{y^{2}} T_{a b}, \\
\partial^{2} \chi_{y a}-\frac{n-2}{y} \partial_{y} \chi_{y a}+\frac{1}{y^{2}}(n-2) \chi_{y a}=-\frac{16 \pi G L^{2}}{y^{2}} T_{y a}, \\
\partial^{2} \tilde{\chi}-\frac{n-2}{y} \partial_{y} \tilde{\chi}+\frac{2}{y^{2}}(n-3) \tilde{\chi}=-\frac{16 \pi G L^{2}}{y^{2}} \tilde{T},
\end{gathered}
$$

where $\tilde{T}:=T^{\rho}{ }_{\rho}-(n-2) T_{y y}$ and the subscript $a, b=0, \ldots$, $n-2$. In this paper we are interested in the $n=4$ dimensional AdS spacetime. In this case, the retarded solutions to (13)-(15) are given by

$$
\begin{aligned}
& \chi_{a b}(x)=16 \pi G \int d^{4} x^{\prime} \frac{L^{2}}{y^{\prime 2}} G_{R}^{\nu=3 / 2}\left(x, x^{\prime}\right) T_{a b}\left(x^{\prime}\right), \\
& \chi_{y a}(x)=16 \pi G \int d^{4} x^{\prime} \frac{L^{2}}{y^{\prime 2}} G_{R}^{\nu=1 / 2}\left(x, x^{\prime}\right) T_{y a}\left(x^{\prime}\right), \\
& \tilde{\chi}(x)=16 \pi G \int d^{4} x^{\prime} \frac{L^{2}}{y^{\prime 2}} G_{R}^{\nu=-1 / 2}\left(x, x^{\prime}\right) \tilde{T}\left(x^{\prime}\right), \\
& \tilde{T}=\eta^{\mu \nu} T_{\mu \nu}-2 T_{y y},
\end{aligned}
$$

where the retarded propagator $G_{R}^{\nu}$ satisfies the differential equations:

$$
\begin{array}{r}
\left(\partial^{2}-\frac{2}{y} \partial_{y}\right) G_{R}^{\nu=3 / 2}\left(x, x^{\prime}\right)=-\frac{y^{2}}{L^{2}} \delta^{(4)}\left(x-x^{\prime}\right), \\
\left(\partial^{2}-\frac{2}{y} \partial_{y}+\frac{2}{y^{2}}\right) G_{R}^{\nu= \pm 1 / 2}\left(x, x^{\prime}\right)=-\frac{y^{2}}{L^{2}} \delta^{(4)}\left(x-x^{\prime}\right) .
\end{array}
$$

Here the retarded propagator in $\mathrm{AdS}_{4}$ with index $\nu$ is given by [30]

$$
\begin{aligned}
G_{R}^{\nu}\left(w, w^{\prime}\right)= & -\frac{\theta\left(t-t^{\prime}-\left|\mathbf{x}-\mathbf{x}^{\prime}\right|\right)}{4 \pi L^{2}} \\
& \times\left[\frac{d}{d z_{+}}\left(\left(\theta\left(1-z_{+}\right)-\theta\left(-1-z_{+}\right)\right) P_{\nu-1 / 2}\left(z_{+}\right)\right)\right. \\
& \left.+2 \cos (\nu \pi) \frac{d}{d z_{-}}\left(\theta\left(z_{-}-1\right) Q_{\nu-1 / 2}\left(z_{-}\right)\right)\right]
\end{aligned}
$$

where $P_{\nu-1 / 2}\left(z_{+}\right)$and $Q_{\nu-1 / 2}\left(z_{-}\right)$are the Legendre functions of the first and second kind respectively, and $z_{ \pm}$are given by

$$
\begin{aligned}
z_{ \pm} & = \pm \frac{-\left(t-t^{\prime}\right)^{2}+\left|\mathbf{x}-\mathbf{x}^{\prime}\right|^{2}+y^{2}+y^{\prime 2}}{2 y y^{\prime}}, \\
\left|\mathbf{x}-\mathbf{x}^{\prime}\right| & =\sqrt{\left(x-x^{\prime}\right)_{i}\left(x-x^{\prime}\right)^{i}} .
\end{aligned}
$$


For $\nu=3 / 2$ and $\nu= \pm 1 / 2, \cos (\nu \pi)=0$ and we get the simple expressions

$$
\begin{aligned}
G_{R}^{\nu=3 / 2}\left(w, w^{\prime}\right)= & \frac{\theta\left(t-t^{\prime}-\left|\mathbf{x}-\mathbf{x}^{\prime}\right|\right)}{2 \pi L^{2}} \\
\times & {\left[y y^{\prime}\left(\delta\left(\left(t-t^{\prime}\right)^{2}-r^{2}\right)+\delta\left(\left(t-t^{\prime}\right)^{2}-\tilde{r}^{2}\right)\right)\right.} \\
- & \left.\frac{1}{2}\left(\theta\left(\left(t-t^{\prime}\right)^{2}-r^{2}\right)-\theta\left(\left(t-t^{\prime}\right)^{2}-\tilde{r}^{2}\right)\right)\right], \\
G_{R}^{\nu= \pm 1 / 2}\left(w, w^{\prime}\right)= & \frac{y y^{\prime} \theta\left(t-t^{\prime}-\left|\mathbf{x}-\mathbf{x}^{\prime}\right|\right)}{2 \pi L^{2}}\left(\delta\left(\left(t-t^{\prime}\right)^{2}-r^{2}\right)\right. \\
& \left.-\delta\left(\left(t-t^{\prime}\right)^{2}-\tilde{r}^{2}\right)\right),
\end{aligned}
$$

where

$r^{2}=\left|\mathbf{x}-\mathbf{x}^{\prime}\right|^{2}+\left(y-y^{\prime}\right)^{2}, \quad \tilde{r}^{2}=\left|\mathbf{x}-\mathbf{x}^{\prime}\right|^{2}+\left(y+y^{\prime}\right)^{2}$.

\section{B. Retarded potential for massless particle scattering}

As a simple example of localized energy-momentum source, let us consider a scattering event of point particles $[7,22]$. The energy-momentum tensor for a particle scattering which occurs at a spacetime point $x_{0}^{\mu}=\left(t_{0}, \mathbf{z}_{0}\right)$ is written as

$$
T_{\mu \nu}=\sum_{j, \text { in }} T_{\mu \nu}^{(j)}+\sum_{n, \text { in }} T_{\mu \nu}^{(n)}+\sum_{i, \text { out }} T_{\mu \nu}^{(i)}+\sum_{m, \text { out }} T_{\mu \nu}^{(m)},
$$

where $T_{\mu \nu}^{(j)}$ is the energy-momentum tensor for the $j$ th incoming massive particle,

$$
\begin{aligned}
T_{\mu \nu}^{(j)} & =M_{\mathrm{in}}^{(j)} u_{\mu}^{(j)} u_{\nu}^{(j)} \delta^{(3)}\left(\mathbf{x}-\mathbf{z}^{(j)}(t)\right) \frac{d \tau^{(j)}}{d t} \frac{\theta\left(t_{0}-t\right)}{\sqrt{-\bar{g}}}, \\
u_{\mu}^{(j)} & =\bar{g}_{\mu \nu} \frac{d x^{\nu(j)}}{d \tau^{(j)}}
\end{aligned}
$$

$T_{\mu \nu}^{(i)}$ is that for the $i$ th outgoing massive particle,

$$
\begin{aligned}
T_{\mu \nu}^{(i)} & =M_{\mathrm{out}}^{(i)} u_{\mu}^{(i)} u_{\nu}^{(i)} \delta^{(3)}\left(\mathbf{x}-\mathbf{z}^{(i)}(t)\right) \frac{d \tau^{(i)}}{d t} \frac{\theta\left(t-t_{0}\right)}{\sqrt{-\bar{g}}}, \\
u_{\mu}^{(i)} & =\bar{g}_{\mu \nu} \frac{d x^{\nu(i)}}{d \tau^{(i)}},
\end{aligned}
$$

$T_{\mu \nu}^{(n)}$ is that for the $n$th incoming massless particle,

$$
\begin{aligned}
T_{\mu \nu}^{(n)} & =k_{\mu}^{(n)} k_{\nu}^{(n)} \delta^{(3)}\left(\mathbf{x}-\mathbf{z}^{(n)}(t)\right) \frac{d \lambda^{(n)}}{d t} \frac{\theta\left(t_{0}-t\right)}{\sqrt{-\bar{g}}}, \\
k_{\mu}^{(n)} & =\bar{g}_{\mu \nu} \frac{d x^{\nu(n)}}{d \lambda^{(n)}},
\end{aligned}
$$

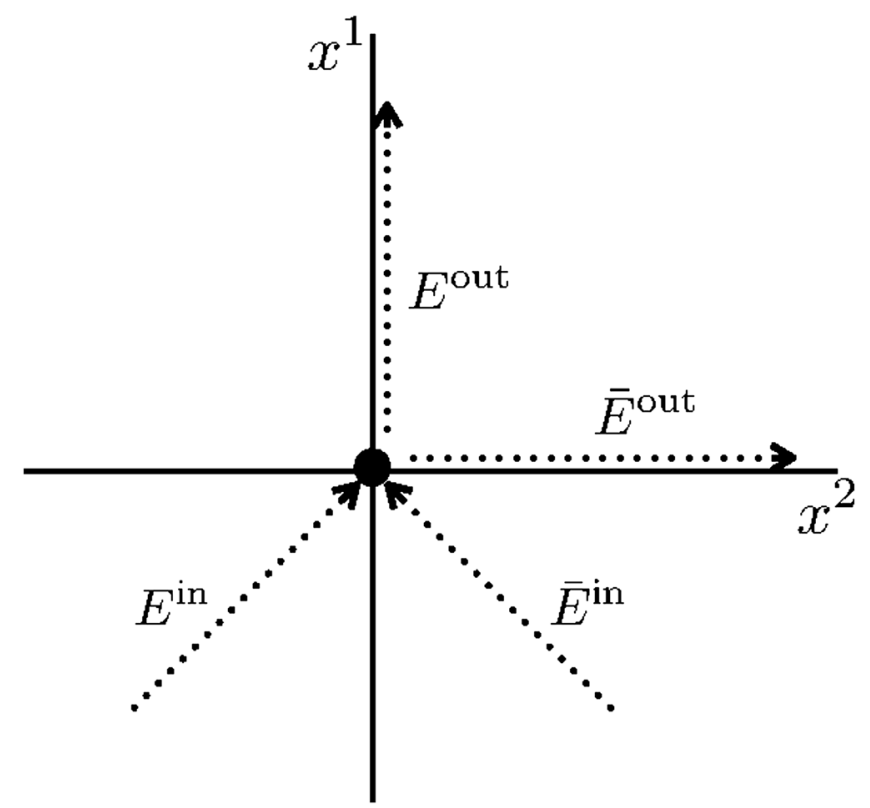

FIG. 1. A massless scattering in the $x^{1}-x^{2}$ plane.

and $T_{\mu \nu}^{(m)}$ is that for the $m$ th outgoing massless particle,

$T_{\mu \nu}^{(m)}=k_{\mu}^{(m)} k_{\nu}^{(m)} \delta^{(3)}\left(\mathbf{x}-\mathbf{z}^{(m)}(t)\right) \frac{d \lambda^{(m)}}{d t} \frac{\theta\left(t-t_{0}\right)}{\sqrt{-\bar{g}}}$,

$k_{\mu}^{(m)}=\bar{g}_{\mu \nu} \frac{d x^{\nu(m)}}{d \lambda^{(m)}}$.

Here $M_{\text {in }}^{(j)}$ and $M_{\text {out }}^{(i)}$ are the rest masses of the incoming and the outgoing massive particles, respectively. $\tau^{(j, i)}$ denotes the proper time of the massive particles while $\lambda^{(n, m)}$ denotes the affine parameter for the null geodesics. $\mathbf{z}^{(j, i, n, m)}(t)$ satisfy $\mathbf{z}^{(j, i, n, m)}\left(t=t_{0}\right)=\mathbf{z}_{0}$. Terms proportional to $\delta\left(t-t_{0}\right)$ in $D_{\mu} T^{\mu}{ }_{\nu}=0$ imply the energymomentum conservation at the interaction point $x_{0}^{\mu}$,

$$
\sum_{j} M_{\mathrm{in}}^{(j)} u_{\mu}^{(j)}+\sum_{n} k_{\mu}^{(n)}=\sum_{i} M_{\mathrm{out}}^{(i)} u_{\mu}^{(i)}+\sum_{\text {out }} k_{\mu}^{(m)} .
$$

In order to evaluate the retarded gravitational potential explicitly, we consider a simple scattering process as shown in Fig. 1, where the scattering takes place in the $x^{1}-x^{2}$ plane with a fixed $y=y_{0}$, where two incoming massless particles collide at $x_{0}^{\mu}=\left(t_{0}, t_{0}, t_{0}, y_{0}\right)$ and results in two outgoing massless particles. By adopting a suitable Lorentz transformation, we can assume without loss of generality that the outgoing particles move along the $x^{1}$ and $x^{2}$ directions orthogonally. For completeness, we list some basic materials about the geodesic motion of a point particle in AdS space in Appendix A.

Note that one can consider more general scattering processes in which both massless and massive particles are involved. In a flat background, one can think of a 
massive particle at rest and its decay into a massive and a massless particle. It would be one of the most simple examples for a particle scattering source which gives rise to both ordinary and null memory due to the emission of the massive and massless particle [7]. In AdS space, evaluation of the retarded potential for a decay of a massive particle that is freely moving along the geodesic could be done explicitly but the final expression would be a complicated one and would not be as illuminating as that for a decay of a massive particle at rest in flat space. On the other hand, geodesic motion of a massless particle in AdS space is as simple as that in flat space and this is the reason why we focus on a massless scattering source here. Although we will not consider the effect of massive particles on the retarded potential in AdS space, we expect that there will be no qualitative differences between the behavior of the retarded potential for massless scattering sources and that for massive sources, and the example investigated in this section would suffice to capture the characteristics of the retarded potential for a particle scattering source.

The energy-momentum tensor for the massless scattering is given by

$$
\begin{aligned}
T_{\mu \nu}(x)= & k_{\mu}^{\text {in }} k_{\nu}^{\text {in }} \delta\left(x^{1}-x_{\text {in }}^{1}\right) \delta\left(x^{2}-x_{\text {in }}^{2}\right) \delta\left(y-y_{0}\right) \frac{d \lambda_{\text {in }}}{d t} \frac{\theta\left(t_{0}-t\right)}{\sqrt{-\bar{g}}} \\
& +\bar{k}_{\mu}^{\text {in }} \bar{k}_{\nu}^{\text {in }} \delta\left(x^{1}-\bar{x}_{\text {in }}^{1}\right) \delta\left(x^{2}-\bar{x}_{\text {in }}^{2}\right) \delta\left(y-y_{0}\right) \frac{d \bar{\lambda}_{\text {in }}}{d t} \frac{\theta\left(t_{0}-t\right)}{\sqrt{-\bar{g}}} \\
& +k_{\mu}^{\text {out }} k_{\nu}^{\text {out }} \delta\left(t-x^{1}\right) \delta\left(x^{2}-t_{0}\right) \delta\left(y-y_{0}\right) \frac{d \lambda_{\text {out }}}{d t} \frac{\theta\left(t-t_{0}\right)}{\sqrt{-\bar{g}}} \\
& +\bar{k}_{\mu}^{\text {out }} \bar{k}_{\nu}^{\text {out }} \delta\left(x^{1}-t_{0}\right) \delta\left(t-x^{2}\right) \delta\left(y-y_{0}\right) \frac{d \bar{\lambda}_{\text {out }}}{d t} \frac{\theta\left(t-t_{0}\right)}{\sqrt{-\bar{g}}}
\end{aligned}
$$

where we set

$$
\begin{aligned}
& x_{\text {in }}^{1}(t)=\frac{1}{\sqrt{2}}\left(t+c_{1}\right), \\
& x_{\text {in }}^{2}(t)=\frac{1}{\sqrt{2}}\left(t+c_{1}\right), \quad c_{1}=(\sqrt{2}-1) t_{0}, \\
& \bar{x}_{\text {in }}^{1}(t)=\frac{1}{\sqrt{2}}\left(t+c_{1}\right), \\
& \bar{x}_{\text {in }}^{2}(t)=-\frac{1}{\sqrt{2}}\left(t+c_{2}\right), \quad c_{2}=-(\sqrt{2}+1) t_{0} .
\end{aligned}
$$

Now we are ready to compute the retarded potential from the outgoing and incoming massless particles. Note that $\chi^{a}{ }_{a}=\chi_{y a}=\tilde{\chi}=0$ for the source (32).

\section{Contribution from outgoing massless particles}

First we consider the contribution from the outgoing massless particles for which the energy-momentum tensor is

$$
\begin{aligned}
T_{\mu \nu}^{\text {out }}(x)= & \frac{y^{2}}{L^{2}} E^{\text {out }} n_{\mu}^{\text {out }} n_{\nu}^{\text {out }} \delta\left(t-x^{1}\right) \delta\left(x^{2}-t_{0}\right) \delta\left(y-y_{0}\right) \theta\left(t-t_{0}\right) \\
& +\frac{y^{2}}{L^{2}} \bar{E}^{\text {out }} \bar{n}_{\mu}^{\text {out }} \bar{n}_{\nu}^{\text {out }} \delta\left(x^{1}-t_{0}\right) \delta\left(t-x^{2}\right) \\
& \times \delta\left(y-y_{0}\right) \theta\left(t-t_{0}\right)
\end{aligned}
$$

where $n_{\mu}^{\text {out }}=-\delta_{\mu}^{0}+\delta_{\mu}^{1}, \bar{n}_{\mu}^{\text {out }}=-\delta_{\mu}^{0}+\delta_{\mu}^{2}$ are the unit tangents to the trajectories of the outgoing massless particles and $E^{\text {out }}, \bar{E}^{\text {out }}$ are the energies of each of the outgoing massless particles.

The retarded potential is evaluated as

$$
\begin{aligned}
\chi_{a b}^{\text {out }}= & \frac{8 G E^{\text {out }}}{L^{2}} n_{a}^{\text {out }} n_{b}^{\text {out }} \int d t^{\prime} \theta\left(t^{\prime}-t_{0}\right) \\
& \times\left[y y_{0}\left(\delta\left(\left(t-t^{\prime}\right)^{2}-r^{2}\right)+\delta\left(\left(t-t^{\prime}\right)^{2}-\tilde{r}^{2}\right)\right)-\frac{1}{2}\left(\theta\left(\left(t-t^{\prime}\right)^{2}-r^{2}\right)-\theta\left(\left(t-t^{\prime}\right)^{2}-\tilde{r}^{2}\right)\right)\right]_{x^{\prime 1}=t^{\prime}, x^{\prime 2}=t_{0}, y^{\prime}=y_{0}} \\
& +\frac{8 G \bar{E}^{\text {out }}}{L^{2}} \bar{n}_{a}^{\text {out }} \bar{n}_{b}^{\text {out }} \int d t^{\prime} \theta\left(t^{\prime}-t_{0}\right)\left[y y_{0}\left(\delta\left(\left(t-t^{\prime}\right)^{2}-r^{2}\right)+\delta\left(\left(t-t^{\prime}\right)^{2}-\tilde{r}^{2}\right)\right)\right. \\
& \left.-\frac{1}{2}\left(\theta\left(\left(t-t^{\prime}\right)^{2}-r^{2}\right)-\theta\left(\left(t-t^{\prime}\right)^{2}-\tilde{r}^{2}\right)\right)\right]_{x^{\prime 1}=t_{0}, x^{\prime 2}=t^{\prime}, y^{\prime}=y_{0}} \\
= & \frac{2 G}{L^{2}}\left(\frac{E^{\text {out }} n_{a}^{\text {out }} n_{b}^{\text {out }}}{t-x^{1}}+\frac{\bar{E}^{\text {out }} \bar{n}_{a}^{\text {out }} \bar{n}_{b}^{\text {out }}}{t-x^{2}}\right)\left[\left(2 y y_{0}-U\right) \theta(u)+\left(2 y y_{0}+\tilde{U}\right) \theta(\tilde{u})\right]
\end{aligned}
$$

where we have used $\theta(U)=\theta(u)$ and $\theta(\tilde{U})=\theta(\tilde{u})$ with

$$
\begin{array}{lll}
u=t-t_{0}-r_{0}, & U=\left(t-t_{0}\right)^{2}-r_{0}^{2}, & r_{0}^{2}=\left(x^{1}-t_{0}\right)^{2}+\left(x^{2}-t_{0}\right)^{2}+\left(y-y_{0}\right)^{2}, \\
\tilde{u}=t-t_{0}-\tilde{r}_{0}, & \tilde{U}=\left(t-t_{0}\right)^{2}-\tilde{r}_{0}^{2}, & \tilde{r}_{0}^{2}=\left(x^{1}-t_{0}\right)^{2}+\left(x^{2}-t_{0}\right)^{2}+\left(y+y_{0}\right)^{2} .
\end{array}
$$




\section{Contribution from incoming massless particles}

Next we consider the retarded potential due to the incoming massless particles. The energy-momentum tensor is

$$
\begin{aligned}
T_{\mu \nu}^{\mathrm{in}}(x)= & \frac{y^{2}}{L^{2}} E^{\mathrm{in}} m_{\mu}^{\mathrm{in}} m_{\nu}^{\mathrm{in}} \delta\left(x^{1}-x_{\mathrm{in}}^{1}\right) \delta\left(x^{2}-x_{\mathrm{in}}^{2}\right) \\
& \times \delta\left(y-y_{0}\right) \theta\left(t_{0}-t\right) \\
& +\frac{y^{2}}{L^{2}} \bar{E}^{\mathrm{in}} \bar{m}_{\mu}^{\mathrm{in}} \bar{m}_{\nu}^{\mathrm{in}} \delta\left(x^{1}-\bar{x}_{\mathrm{in}}^{1}\right) \delta\left(x^{2}-\bar{x}_{\mathrm{in}}^{2}\right) \\
& \times \delta\left(y-y_{0}\right) \theta\left(t_{0}-t\right),
\end{aligned}
$$

where $m_{\mu}^{\text {in }}=-\delta_{\mu}^{0}+\left(\delta_{\mu}^{1}+\delta_{\mu}^{2}\right) / \sqrt{2}, \bar{m}_{\mu}^{\text {in }}=-\delta_{\mu}^{0}+\left(\delta_{\mu}^{1}-\delta_{\mu}^{2}\right) / \sqrt{2}$ are the unit tangents to the trajectories of the incoming massless particles. The energy-momentum conservation (31) at $t=t_{0}$ is solved by

$$
E^{\text {in }}=E^{\text {out }}, \quad \bar{E}^{\text {in }}=\bar{E}^{\text {out }}=(\sqrt{2}-1) E^{\text {in }} .
$$

The retarded potential due to the incoming massless particles is given by

$$
\begin{aligned}
& \chi_{a b}^{\text {in }}=\frac{4 G y y_{0}}{L^{2}}\left(\frac{E^{\mathrm{in}} m_{a}^{\mathrm{in}} m_{b}^{\mathrm{in}}}{t+c_{1}-\frac{x^{1}+x^{2}}{\sqrt{2}}}+\frac{\bar{E}^{\mathrm{in}} \bar{m}_{a}^{\mathrm{in}} \bar{m}_{b}^{\mathrm{in}}}{t-t_{0}-\frac{x^{1}-x^{2}}{\sqrt{2}}}\right)(\theta(-u)+\theta(-\tilde{u})) \\
& -\frac{4 G}{L^{2}}\left[E^{\mathrm{in}} m_{a}^{\mathrm{in}} m_{b}^{\mathrm{in}}\left(\theta(-u) \int_{t_{c}}^{t_{-}} d t^{\prime}-\theta(-\tilde{u}) \int_{t_{c}}^{t_{+}} d t^{\prime}+(\theta(u)-\theta(\tilde{u})) \int_{t_{c}}^{t_{0}} d t^{\prime}\right)\right. \\
& \left.+\bar{E}^{\mathrm{in}} \bar{m}_{a}^{\mathrm{in}} \bar{m}_{b}^{\mathrm{in}}\left(\theta(-u) \int_{t_{c}}^{\bar{t}_{-}} d t^{\prime}-\theta(-\tilde{u}) \int_{t_{c}}^{\bar{t}_{+}} d t^{\prime}+(\theta(u)-\theta(\tilde{u})) \int_{t_{c}}^{t_{0}} d t^{\prime}\right)\right] \\
& =\frac{4 G y y_{0}}{L^{2}}\left(\frac{E^{\text {in }} m_{a}^{\text {in }} m_{b}^{\text {in }}}{t+c_{1}-\frac{x^{1}+x^{2}}{\sqrt{2}}}+\frac{\bar{E}^{\text {in }} \bar{m}_{a}^{\text {in }} \bar{m}_{b}^{\text {in }}}{t-t_{0}-\frac{x^{1}-x^{2}}{\sqrt{2}}}\right)(\theta(-u)+\theta(-\tilde{u})) \\
& -\frac{4 G}{L^{2}}\left[E^{\mathrm{in}} m_{a}^{\mathrm{in}} m_{b}^{\mathrm{in}}\left(\theta(-u) t_{-}-\theta(-\tilde{u}) t_{+}+(\theta(u)-\theta(\tilde{u})) t_{0}\right)\right. \\
& \left.+\bar{E}^{\text {in }} \bar{m}_{a}^{\text {in }} \bar{m}_{b}^{\text {in }}\left(\theta(-u) \bar{t}_{-}-\theta(-\tilde{u}) \bar{t}_{+}+(\theta(u)-\theta(\tilde{u})) t_{0}\right)\right], \\
& =\frac{2 G}{L^{2}}\left(\frac{E^{\text {in }} m_{a}^{\text {in }} m_{b}^{\text {in }}}{t+c_{1}-\frac{x^{1}+x^{2}}{\sqrt{2}}}+\frac{\bar{E}^{\text {in }} \bar{m}_{a}^{\text {in }} \bar{m}_{b}^{\text {in }}}{t-t_{0}-\frac{x^{1}-x^{2}}{\sqrt{2}}}\right)\left[\left(2 y y_{0}-U\right) \theta(-u)+\left(2 y y_{0}+\tilde{U}\right) \theta(-\tilde{u})\right],
\end{aligned}
$$

where $t_{c}$ is an infrared cutoff whose dependence cancels out in the end. $t_{\mp}$ and $\bar{t}_{\mp}$ are the solution to $\left(t-t_{-}\right)^{2}-$ $r^{2}\left(t_{-}\right)=0,\left(t-t_{+}\right)^{2}-\tilde{r}^{2}\left(t_{+}\right)=0$ for the unbarred null geodesic and $\left(t-\bar{t}_{-}\right)^{2}-r^{2}\left(\bar{t}_{-}\right)=0,\left(t-\bar{t}_{+}\right)^{2}-\tilde{r}^{2}\left(\bar{t}_{+}\right)=0$ for the barred null geodesic. Explicitly, it is

$$
\begin{aligned}
& t_{\mp}=\frac{t^{2}-x^{1}\left(x^{1}-\sqrt{2} c_{1}\right)-x^{2}\left(x^{2}-\sqrt{2} c_{1}\right)-\left(y \mp y_{0}\right)^{2}-c_{1}^{2}}{2\left(t+c_{1}-\frac{x^{1}+x^{2}}{\sqrt{2}}\right)}, \\
& \bar{t}_{\mp}=\frac{t^{2}-x^{1}\left(x^{1}-\sqrt{2} c_{1}\right)-x^{2}\left(x^{2}-\sqrt{2} c_{2}\right)-\left(y \mp y_{0}\right)^{2}-3 t_{0}^{2}}{2\left(t-t_{0}-\frac{x^{1}-x^{2}}{\sqrt{2}}\right)} .
\end{aligned}
$$

\section{Behavior of the retarded potential}

Putting together (35) and (40) and making use of the identity

$$
\begin{aligned}
& {\left[\left(2 y y_{0}-U\right) \theta(u)+\left(2 y y_{0}+\tilde{U}\right) \theta(\tilde{u})\right]} \\
& \quad+\left[\left(2 y y_{0}-U\right) \theta(-u)+\left(2 y y_{0}+\tilde{U}\right) \theta(-\tilde{u})\right]=0,
\end{aligned}
$$

the retarded potential for the massless scattering source $\chi=\chi^{\text {out }}+\chi^{\text {in }}$ is obtained as

$$
\begin{aligned}
& \chi_{a b}=\frac{2 G}{L^{2}}\left(\alpha_{a b}-\beta_{a b}\right)\left[\left(2 y y_{0}-U\right) \theta(u)+\left(2 y y_{0}+\tilde{U}\right) \theta(\tilde{u})\right], \\
& \chi_{y a}=0, \quad \tilde{\chi}=0,
\end{aligned}
$$

where we have defined $\alpha_{a b}$ and $\beta_{a b}$ by

$$
\begin{aligned}
& \alpha_{a b}:=\frac{E^{\text {out }} n_{a}^{\text {out }} n_{b}^{\text {out }}}{t-x^{1}}+\frac{\bar{E}^{\text {out }} \bar{n}_{a}^{\text {out }} \bar{n}_{b}^{\text {out }}}{t-x^{2}}, \\
& \beta_{a b}:=\frac{E^{\text {in }} m_{a}^{\text {in }} m_{b}^{\text {in }}}{t+c_{1}-\frac{x^{1}+x^{2}}{\sqrt{2}}}+\frac{\bar{E}^{\text {in }} \bar{m}_{a}^{\text {in }} \bar{m}_{b}^{\text {in }}}{t-t_{0}-\frac{x^{1}-x^{2}}{\sqrt{2}}} .
\end{aligned}
$$

The result (43) gives the original metric perturbation $\gamma_{\mu \nu}{ }^{3}$ :

\footnotetext{
${ }^{3}$ The gauge condition (8) in this case reads $\partial_{a} \gamma^{a}{ }_{b}=0$. This implies $\left(\alpha_{a b}-\beta_{a b}\right) K^{a} \delta(u)=0$ and $\left(\alpha_{a b}-\beta_{a b}\right) \tilde{K}^{a} \delta(\tilde{u})=0$, respectively, where $K^{a}=\partial^{a} u=-\left(t^{a}+r_{0}^{a}\right)$ and $\tilde{K}^{a}=\partial^{a} \tilde{u}=$ $-\left(t^{a}+\tilde{r}_{0}^{a}\right)$.
} 


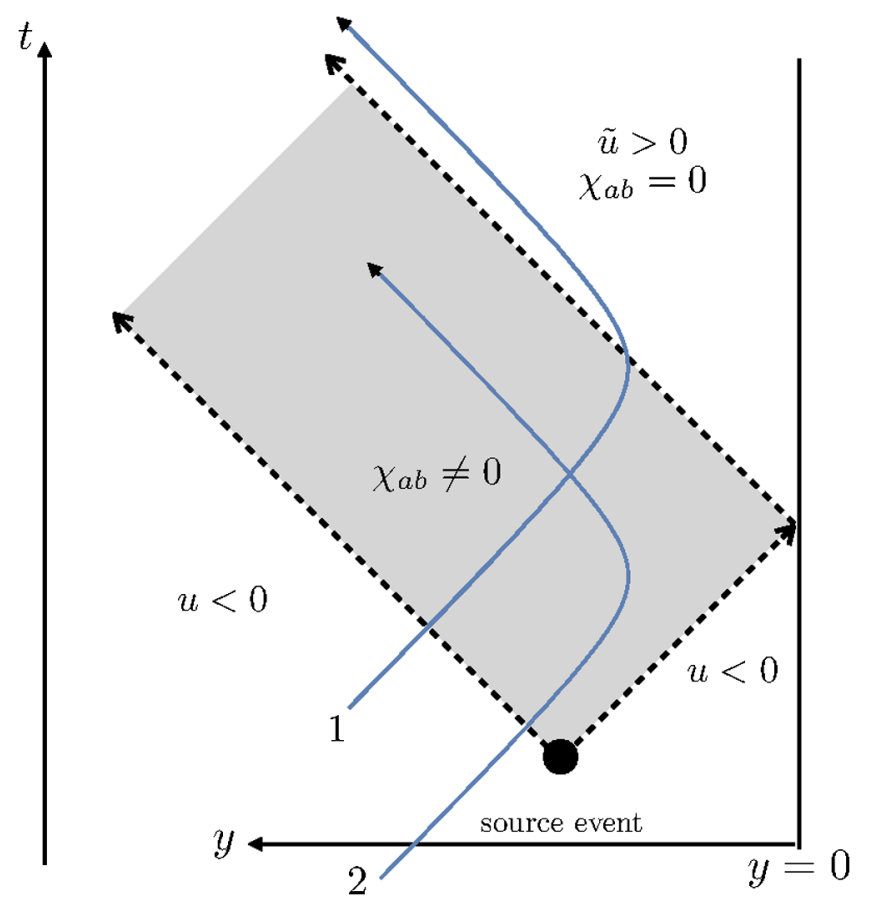

FIG. 2. Propagation of the gravitational waves and timelike geodesics.

$$
\gamma_{a b}=\frac{L^{2}}{y^{2}} \chi_{a b}, \quad \gamma_{y a}=0, \quad \gamma_{y y}=0
$$

We note that in (43), the terms proportional to $U$ and $\tilde{U}$ are the tail contribution. The ratio of the direct and tail contributions of the gravitational waves which directly come from the source event is thus

$$
\frac{\text { tail }}{\text { direct }} \approx \frac{r_{0} \Delta t}{y y_{0}}
$$

where $\Delta t:=t-t_{0}-r_{0}$ measures the time passed since the passage of gravitational waves.

Figure 2 shows a two-dimensional spacetime diagram for the behavior of the retarded potential $\chi_{a b}$ where the $x^{i}$ directions are suppressed. The bullet represents the source event, the dotted lines represent the propagation of gravitational waves traveling at the speed of light. Gravitational waves and their tails reach the AdS boundary in a finite time and then will get reflected back to the bulk.

Let us consider two timelike observers whose geodesics are given by

$$
\begin{aligned}
y^{2}\left(\tau_{1,2}\right) & =\left(t\left(\tau_{1,2}\right)-T_{1,2}\right)^{2}+L^{2}, \\
x^{1} & =\text { const }, \quad x^{2}=\text { const },
\end{aligned}
$$

where $\tau_{1,2}$ are the proper times and $T_{1,2}$ are constants with $T_{2}<t_{0}<T_{1}$ for the observers 1 and 2 , respectively. The worldlines of these observers are represented by the solid curves in Fig. 2. The retarded potential $\chi_{a b}$ for these observers acquires a nonzero contribution discontinuously on the light cone $t=t_{0}+r$ due to the direct contribution. In the region $t_{0}+r<t<t_{0}+\tilde{r}$, the tail contribution is nonzero and reduces the effect of the potential $\chi_{a b}$. When $t \approx t_{r}+y y_{0} / r_{0}$, the tail contribution becomes comparable to the direct contribution. Once $t=t_{0}+\tilde{r}$, which happens to observer 1 , then $\chi_{a b}$ vanishes discontinuously as the second term in (35) which is proportional to $\theta(\tilde{u})$ comes into action. This means that the contribution from the reflected gravitational waves cancels exactly that from those directly from the source and the spacetime goes back to the original vacuum AdS space. It follows that $\chi_{a b}$ is nonzero only in the gray region in between the two light cones. Note that for the observer $2, t \geq t_{0}+\tilde{r}$ will never be realized and the cancellation does not occur.

\section{MEMORY EFFECT IN AdS SPACE}

In flat space, the memory effect is defined as a permanent displacement in the geodesic deviation of a pair of test particles after the passage of gravitational waves. In this paper, we will use this observable to give a definition for the memory effect in AdS space. The geodesic deviation equation is

$$
u^{\rho} \nabla_{\rho}\left(u^{\sigma} \nabla_{\sigma} D^{\mu}\right)=-R_{\alpha \beta \gamma}^{\mu} u^{\alpha} D^{\beta} u^{\gamma}
$$

Here we consider two nearby test particles initially at rest as a gravitational wave detector. $D^{\mu}$ is the deviation vector of the test particles and $u^{\mu}=d x^{\mu} / d \tau$ is the unit tangent to the geodesic of one of the test particles. In what follows, we will call this geodesic the central geodesic $\gamma$ which is, at the zeroth order of the perturbation, parametrized by the proper time $\tau(-\pi L / 2<\tau<\pi L / 2)$ as

$y(\tau)=\frac{L}{\cos \left(\frac{\tau}{L}\right)}, \quad t(\tau)=L \tan \left(\frac{\tau}{L}\right), \quad x^{1}=x^{2}=\mathrm{const}$

with $y^{2}(\tau)=t^{2}(\tau)+L^{2}$.

In general, the geodesic deviation

$$
D^{\mu}=\bar{D}^{\mu}+\delta D^{\mu}
$$

gets an intrinsic contribution $\bar{D}^{\mu}$ from the curvature of the background spacetime and this is independent of the contribution $\delta D^{\mu}$ from the gravitational wave that we are interested in. If one subtracts out the contribution from the background curvature, one can obtain from (48) a second order differential equation for $\delta D^{\mu}$. This can be solved in principle and will give generally an integrated expression for $\delta D^{\mu}$ in terms of the full history of the metric perturbation. This is much more complicated than the local expression (1) obtained in the flat case. In the following, we show that a simple local expression can be obtained if the memory $\delta D^{\mu}$ is observed with respect to a certain local 
inertial frame, the Fermi normal coordinate, around the central geodesic $\gamma$.

\section{A. Gravitational radiation induced geodesic derivation}

In order to simplify the study of the geodesic derivation, it is desirable to adopt a coordinate system that is somehow adapted to the central geodesic. In this regard, we find it convenient to use the Fermi normal coordinates (FNC) [31] associated with the central geodesic $\gamma(\tau)$, $x_{F}^{\alpha}=\left(t_{F}=\tau, x_{F}^{r}\right), r=1,2,3$, where the time coordinate is identified with the proper time and the spatial coordinates $x_{F}^{r}$ are parametrized by the affine parameter of geodesics perpendicular to the central geodesic $\gamma(\tau)$ on which $x_{F}^{\alpha}=(\tau, 0,0,0)$. In the following, the subscript $F$ stands for quantity in the FNC. The Fermi normal coordinate system is a locally flat coordinate system attached to the central geodesic, i.e., on the central geodesic $\gamma(\tau)$, the metric is given by Minkowski metric $g_{\mu \nu}^{F}=\eta_{\mu \nu}$ and the Christoffel symbols vanish, $\left(\Gamma_{F}\right)_{\alpha \beta}^{\mu}=0$. In the neighborhood of the central geodesic, the metric receives corrections from the spacetime curvature which begins with the quadratic term in $x_{F}^{r}$ of the form $\left(R_{F}\right)_{\mu r u s} x_{F}^{r} x_{F}^{s}$. In AdS space, $\left|R_{F}\right| \simeq L^{-2}$ and the use of FNC at a spacetime point $P$ away from the central geodesic is valid as long as $\left(x_{F}^{r}\right)^{2}(P) \ll L^{2}$.

In the FNC, the tangent to the geodesic is $u_{F}^{\mu}=$ $d x_{F}^{\mu} / d \tau=\delta_{0}^{\mu}$. Then the geodesic deviation equation takes the form

$$
\frac{d^{2}}{d \tau^{2}} D_{F}^{r}=-\left(R_{F}\right)^{r}{ }_{0 s 0}(\tau) D_{F}^{s},
$$

where $\left(R_{F}\right)^{\mu}{ }_{\alpha \beta \gamma}(\tau)$ is the Riemann tensor in the FNC that is evaluated on $\gamma(\tau)$ and it is related to the Riemann tensor in the Poincaré coordinates by

$$
\left(R_{F}\right)^{\mu}{ }_{\alpha \beta \gamma}(\tau)=\eta^{\mu \delta} R_{\nu \rho \sigma \lambda}\left(e_{\delta}\right)^{\nu}\left(e_{\alpha}\right)^{\rho}\left(e_{\beta}\right)^{\sigma}\left(e_{\gamma}\right)^{\lambda} .
$$

Here $\left(e_{\delta}\right)^{\nu}$ is a set of the orthonormal tetrads which is parallel transported along $\gamma(\tau)$ and satisfies [33]

$$
\begin{aligned}
\left(e_{\alpha}\right)^{\mu} & =\left(\frac{\partial x^{\mu}}{\partial x_{F}^{\alpha}}\right)_{\gamma(\tau)}, \frac{d}{d \tau}\left(e_{\alpha}\right)^{\mu}+\Gamma_{\rho \sigma}^{\mu}\left(e_{0}\right)^{\rho}\left(e_{\alpha}\right)^{\sigma}=0, \\
g_{\mu \nu}\left(e_{\alpha}\right)^{\mu}\left(e_{\beta}\right)^{\nu} & =\eta_{\alpha \beta},
\end{aligned}
$$

where $\left(e_{0}\right)^{\mu}$ is taken as the tangent to $\gamma(\tau),\left(e_{0}\right)^{\mu}=u^{\mu}$. For the background AdS space, the orthonormal tetrads on (49) are determined as

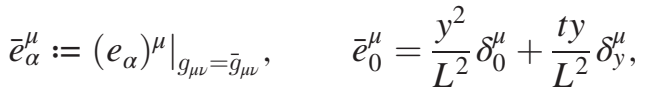

$\bar{e}_{i}^{\mu}=\frac{y}{L} \delta_{i}^{\mu}, \quad \bar{e}_{3}^{\mu}=-\frac{t y}{L^{2}} \delta_{0}^{\mu}-\frac{y^{2}}{L^{2}} \delta_{y}^{\mu}$.

The geodesic and parallel transport equations in the perturbative AdS background are discussed in Appendix C.

At the first order of the perturbation $\chi_{a b}$ given by (43), we obtain

$$
\begin{aligned}
\left(R_{F}\right)_{r 0 s 0}= & \frac{1}{L^{2}} \eta_{r s}+\frac{1}{2 L^{4}} \delta_{r}^{i} \delta_{s}^{j}\left[y^{4}\left(2 \partial_{0} \partial_{(j} \chi_{i) 0}-\partial_{0}^{2} \chi_{i j}-\partial_{i} \partial_{j} \chi_{00}\right)+2 t y^{3}\left(\partial_{y} \partial_{(j} \chi_{i) 0}-\partial_{0} \partial_{y} \chi_{i j}\right)-t^{2} y^{2} \partial_{y}^{2} \chi_{i j}+y^{3} \eta_{i j} \partial_{y} \chi_{00}-y L^{2} \partial_{y} \chi_{i j}\right] \\
& -\frac{1}{2 L^{5}}\left(\delta_{r}^{i} \delta_{s}^{3}+\delta_{r}^{3} \delta_{s}^{i}\right)\left[y^{3} L^{2}\left(\partial_{y} \partial_{0} \chi_{i 0}-\partial_{y} \partial_{i} \chi_{00}\right)+t y^{2} L^{2}\left(\partial_{y}^{2} \chi_{i 0}-\frac{1}{y} \partial_{y} \chi_{0 i}\right)\right] \\
& +\frac{y^{2}}{2 L^{2}} \delta_{r}^{3} \delta_{s}^{3}\left(-\partial_{y}^{2} \chi_{00}+\frac{1}{y} \partial_{y} \chi_{00}\right)
\end{aligned}
$$

In deriving this, we have used (B2) in Appendix B, (C1), (C7) and (C8) in Appendix C together with (52). It is remarkable that the perturbations of the geodesic $\delta x^{\mu}$ and the tetrads $\delta e_{\alpha}^{\mu}$ do not appear in the final result. Note that the first line in (55) arises from the background curvature in the FNC and gives an oscillating solution for the background geodesic deviation. Below we will focus on the deviation

\footnotetext{
${ }^{4}$ In dS space, due to the exponential expansion of the physical distance between two nearby geodesics, the use of FNC for the analysis of the geodesic deviation equation will be invalidated at late times. In such a case, instead of FNC, one should use the conformal Fermi coordinates [32].
}

vector with an initial condition $d \bar{D}_{F}^{\mu} / d \tau\left(\tau_{i}\right)=0$. In this case we have

$$
\bar{D}_{F}^{r}=C^{r} \cos \left(\frac{\tau-\tau_{i}}{L}\right)
$$

where $C^{r}$ is the initial separation. Note that we have explicitly isolated the geodesic derivation (56) due to the AdS background curvature.

Since the Riemann tensor (55) has a complicated form, it may appear not easy to solve the geodesic deviation equation (51) which is a second order differential equation in the perturbed AdS spacetime. However there is a trick. 
Let us introduce a tensor defined by ${\Omega^{\mu}}_{\nu}:=\nabla_{\nu}\left(e_{0}\right)^{\mu}$. It has been demonstrated recently in [34], especially for shockwave metrics of Aichelburg-Sexl type [35], that the tensor $\Omega^{\mu}{ }_{\nu}$ are useful for the investigation of the memory effect. In terms of $\Omega^{\mu}{ }_{\nu}$, the Riemann tensor can be expressed as

$$
\frac{d}{d \tau}{\Omega^{\mu}}_{\nu}+{\Omega^{\mu}}_{\lambda} \Omega^{\lambda}{ }_{\nu}=-R_{\alpha \nu \beta}^{\mu}\left(e_{0}\right)^{\alpha}\left(e_{0}\right)^{\beta}
$$

and the deviation vector $D^{\mu}$ satisfies the first order differential equation [36],

$$
\left(e_{0}\right)^{\rho} \nabla_{\rho} D^{\mu}=\Omega_{\nu}^{\mu} D^{\nu} .
$$

In the $\mathrm{FNC}$, we have

$$
\frac{d}{d \tau}\left(\Omega_{F}\right)_{\nu}^{\mu}+\left(\Omega_{F}\right)_{\lambda}^{\mu}\left(\Omega_{F}\right)_{\nu}^{\lambda}=-\left(R_{F}\right)^{\mu}{ }_{0 \nu 0},
$$

and

$$
\frac{d}{d \tau} D_{F}^{\mu}=\left(\Omega_{F}\right)_{\nu}^{\mu} D_{F}^{\nu}
$$

Thus the introduction of $\Omega^{\mu}{ }_{\nu}$ and the employment of FNC allow us to greatly reduce the problem of determining $D_{\mu}$ from solving second order partial differential equations to solving a first order ordinary differential equation.

To proceed, let us compute $\left(\Omega_{F}\right)^{\mu}{ }_{\nu}$ :

$$
\left(\Omega_{F}\right)^{\mu}{ }_{\nu}=\eta^{\mu \sigma} g_{\alpha \lambda}\left(e_{\sigma}\right)^{\lambda}\left(e_{\nu}\right)^{\beta} \nabla_{\beta}\left(e_{0}\right)^{\alpha} .
$$

The spatial components of (61) for the central geodesic $\gamma(\tau)$ in the perturbed AdS background is given by

$$
\left(\Omega_{F}\right)_{s}^{r}=\left(\bar{\Omega}_{F}\right)^{r}+\left(\delta \Omega_{F}\right)^{r},
$$

where

$$
\begin{aligned}
\left(\bar{\Omega}_{F}\right)^{r}= & -\frac{t}{L^{2}} \delta^{r}, \\
\left(\delta \Omega_{F}\right)^{r}= & \frac{1}{y}\left(\frac{t}{L^{2}} \delta x^{y}-\delta e_{0}^{y}\right) \delta^{r} \\
& +\frac{1}{2} \eta^{r p} \bar{g}_{\alpha \lambda} \bar{e}_{p}^{\lambda}\left(\partial_{\beta} h^{\alpha}{ }_{\gamma}+\partial_{\gamma} h^{\alpha}{ }_{\beta}-\partial^{\alpha} h_{\beta \gamma}\right) \bar{e}_{s}^{\beta} \bar{e}_{0}^{\gamma} \\
& +\frac{2}{y} \eta^{r p} h_{0 \lambda} \bar{e}_{s}^{y} \bar{e}_{p}^{\lambda}+\eta^{r p} \bar{g}_{\alpha \lambda}\left(\delta e_{p}^{\lambda} \bar{e}_{s}^{\beta} \partial_{\beta} \bar{e}_{0}^{\alpha}+\bar{e}_{p}^{\lambda} \bar{e}_{s}^{\beta} \partial_{\beta} \delta e_{0}^{\alpha}\right),
\end{aligned}
$$

where $h_{\mu \nu}=y^{2} L^{-2} \gamma_{\mu \nu}$. Putting $D_{F}^{r}=\bar{D}_{F}^{r}+\delta D_{F}^{r}$ into (60), the geodesic derivation vector that arises from the perturbation of the AdS metric satisfies

$$
\frac{d}{d \tau}\left(\frac{y}{L} \delta D_{F}^{r}\right)=\frac{y}{L}\left(\delta \Omega_{F}\right)^{r} \bar{D}_{F}^{s}+O\left(\delta D_{F}^{2}\right)
$$

where $\bar{D}_{F}^{r}$ is given by (56).

Now we are to solve the geodesic deviation equation (64) with the metric perturbation given by the retarded potential (43). Consider the specific example of

$$
x^{1}=x^{2}=t_{0}
$$

for the central geodesic $\gamma(\tau)$. Applying (65) to (43), we find that $\chi_{0 a}=0$ and $f^{\mu}$ given by (C6) vanishes. It then follows from (C10) [with a set of initial conditions $\delta x^{\mu}\left(\tau_{i}\right)=$ $\left.d \delta x^{\mu} / d \tau\left(\tau_{i}\right)=0\right]$ that $\delta x^{\mu}=\delta e_{0}^{\mu}=0$. Therefore (49) with (65) is indeed a consistent solution to the perturbed geodesic equation. As a result, we get

$$
\left(\delta \Omega_{F}\right)^{r}{ }_{s}=\frac{1}{2} \frac{d}{d \tau} \chi^{i}{ }_{j} \delta^{r}{ }_{i} \delta_{s}^{j}, \quad\left(\delta \Omega_{F}\right)^{r}{ }_{r}=0
$$

and the geodesic equation (64) can be integrated to a closed form immediately, giving

$$
\begin{aligned}
& D_{F}^{i}(\tau)=\bar{D}_{F}^{i}(\tau)+\frac{1}{2} \chi^{i}{ }_{j}(\tau) \bar{D}_{F}^{j}(\tau) \quad(i, j=1,2), \\
& D_{F}^{3}(\tau)=\bar{D}_{F}^{3}(\tau) .
\end{aligned}
$$

It is easy to check that (67) satisfies the geodesic deviation equation (51) in this case. The geodesic deviation in the Poincaré coordinates is given by $D^{\mu}=\left(e_{\alpha}\right)^{\mu} D_{F}^{\alpha}$.

It is remarkable that the perturbation of the deviation vector (67) can be written in terms of the retarded potential directly and takes the form (1) exactly as in the flat case. That this is possible is because of the adaptation and simplification brought about by the use of the Fermi normal coordinates.

\section{B. Gravitational radiation memory in AdS}

Memory effect as a permanent displacement of the geodesic derivation can be defined in the FNC by

$$
\Delta D_{F}^{r}:=\delta D_{F}^{r}\left(\tau_{f}\right)-\delta D_{F}^{r}\left(\tau_{i}\right),
$$

where $\tau_{i}$ and $\tau_{f}$ are the proper time before and after the passage of gravitational waves.

We have ignored the background effect on the geodesic deviation since we are interested in the geodesic deviation with gravitational wave origin. In AdS spacetime, two distinguished types of gravitational wave detectors can be considered. One is a detector whose central geodesic passes through the region of nonzero retarded potential, e.g., observer 1 in Fig. 2. The other is a detector whose central geodesic stays in the region of nonzero retarded potential, e.g., observer 2 in Fig. 2 . In the former case $\left(t_{0}<0\right)$, we can discuss the memory effect from a viewpoint of a vacuum to vacuum transition of the spacetime. But now it is 
obvious that there is no memory effect since after the passage of the reflected gravitational waves the spacetime settles down to the original vacuum AdS space described by (5) due to the cancellation of the retarded potential. In the latter case $\left(t_{0}>0\right)$, the gravitational wave detector will always be under the influence of the retarded potential since the passage of the direct gravitational waves from the source, and there will be the competition between the direct and the tail contributions. From (43), the retarded potential for the central geodesic (49) with $x^{1}=x^{2}=t_{0}$ is

$$
\begin{aligned}
\chi_{i j} & =a_{i j} \frac{2 G}{L^{2}} \frac{E^{\text {out }}}{t-t_{0}}\left(2 y y_{0}-\left(t-t_{0}\right)^{2}+\left(y-y_{0}\right)^{2}\right) \theta(u) \\
& =a_{i j} \frac{2 G}{L^{2}} \frac{E^{\text {out }}}{t-t_{0}}\left(2 t t_{0}+L^{2}-t_{0}^{2}+y_{0}^{2}\right) \theta(u),
\end{aligned}
$$

where $a_{i j}:=(1-\sqrt{2} / 2)\left(\delta_{i}^{1} \delta_{j}^{1}-\delta_{i}^{1} \delta_{j}^{2}-\delta_{i}^{2} \delta_{j}^{1}-\delta_{i}^{2} \delta_{j}^{2}\right)$. Here we have used $y^{2}=t^{2}+L^{2}$ and have dropped the term proportional to $\theta(\tilde{u})$. It can be seen that at late times $\chi_{i j}$ will approach a constant value

$$
\chi_{i j} \sim a_{i j} \frac{4 G t_{0}}{L} .
$$

Therefore the geodesic deviation will also approach some constant value and yields a permanent displacement $\Delta D_{F}^{r} \neq 0$ while the metric satisfies the vacuum Einstein equation in AdS space.

\section{Asymptotic expansion near the AdS boundary}

The asymptotic behavior of the retarded potential near the AdS boundary can be obtained by the Taylor expansion of (43) at $y \rightarrow 0$. Using

$$
\begin{aligned}
& \left(2 y y_{0}-U\right) \theta(U) \\
& =-U_{0} \theta\left(U_{0}\right)+\left[\theta\left(U_{0}\right)+U_{0}\left(\delta\left(U_{0}\right)-2 y_{0}^{2} \delta^{\prime}\left(U_{0}\right)\right)\right] y^{2} \\
& +2 y_{0}\left[\delta\left(U_{0}\right)+U_{0}\left(\delta^{\prime}\left(U_{0}\right)-\frac{2 y_{0}^{2}}{3} \delta^{\prime \prime}\left(U_{0}\right)\right)\right] y^{3}+\mathcal{O}\left(y^{4}\right) \text {, } \\
& \left(2 y y_{0}+\tilde{U}\right) \theta(\tilde{U}) \\
& =U_{0} \theta\left(U_{0}\right)-\left[\theta\left(U_{0}\right)+U_{0}\left(\delta\left(U_{0}\right)-2 y_{0}^{2} \delta^{\prime}\left(U_{0}\right)\right)\right] y^{2} \\
& +2 y_{0}\left[\delta\left(U_{0}\right)+U_{0}\left(\delta^{\prime}\left(U_{0}\right)-\frac{2 y_{0}^{2}}{3} \delta^{\prime \prime}\left(U_{0}\right)\right)\right] y^{3}+\mathcal{O}\left(y^{4}\right),
\end{aligned}
$$

where $U_{0}:=\left(t-t_{0}\right)^{2}-\left(x^{1}-t_{0}\right)^{2}-\left(x^{2}-t_{0}\right)^{2}-y_{0}^{2}$, we obtain

$\chi_{a b}=\frac{4 G\left(y y_{0}\right)^{3}}{3 L^{2} r_{\mathrm{B}}^{3}} \delta\left(u_{0}\right)\left(1-r_{\mathrm{B}} \frac{\partial}{\partial t}\right)\left(\alpha_{a b}-\beta_{a b}\right)+\mathcal{O}\left(y^{5}\right)$, where $u_{0}=t-t_{0}-r_{\mathrm{B}}$ and

$$
r_{\mathrm{B}}=\sqrt{\left(x^{1}-t_{0}\right)^{2}+\left(x^{2}-t_{0}\right)^{2}+y_{0}^{2}}
$$

is the distance from the scattering event to a point under consideration at the boundary. We note there is a delta function singularity localized at the light cone from the source and it arises because of a careful handling of the step functions as distribution.

The vacuum expectation value of boundary energymomentum tensor is given by the formula [37]

$$
\left\langle T_{a b}^{\mathrm{B}}\right\rangle=\frac{3 L^{2}}{16 \pi G} \lim _{y \rightarrow 0} \frac{1}{y^{3}} \chi_{a b} .
$$

Substituting (73) into (75) yields

$$
\left\langle T_{a b}^{\mathrm{B}}\right\rangle=\frac{y_{0}^{3}}{4 \pi r_{\mathrm{B}}^{3}} \delta\left(u_{0}\right)\left(1-r_{\mathrm{B}} \frac{\partial}{\partial t}\right)\left(\alpha_{a b}-\beta_{a b}\right) .
$$

Thus the boundary energy-momentum tensor is localized on the hyperbola $u_{0}=0$.

\section{AdS shock wave}

In addition to displacement memory, gravitational wave may induce other observable effects on a detector. In the flat case, a notable effect is that a shock wave would give rise to a velocity memory in the form of a relative velocity kick between two nearby particles. It is interesting to know if a shock wave in AdS spacetime would give rise to a velocity memory; and if so, how different would it be from the flat case?

Let us consider the shock-wave limit of the retarded potential. Naively if we take $t_{0} \rightarrow-\infty$ in (32), the source describes massless particles which travel at the speed of light forever. In flat space, the gravitational field of such a massless particle is described by the Aichelburg-Sexl shock-wave metric [35] which is localized on the light cone. In AdS space, such a shock-wave metric was first obtained in [38]. Note that, however, the AdS shock-wave metric cannot be obtained by simply taking $t_{0} \rightarrow-\infty$ in the retarded solution (35) since it vanishes for $t_{0} \rightarrow-\infty$. Instead, following [39], we start with a massless source with a finite extent $\Delta$ in the $x^{1}$ direction with the energymomentum tensor given by

$$
\begin{aligned}
T_{\mu \nu}^{\mathrm{sw}}(x)= & \frac{y^{2}}{L^{2}} E_{\mu} n_{\nu} \frac{1}{\Delta}\left[\theta\left(t-x^{1}\right)-\theta\left(t-x^{1}-\Delta\right)\right] \\
& \times \delta\left(x^{2}\right) \delta\left(y-y_{0}\right) \theta\left(x^{1}-t_{0}\right) .
\end{aligned}
$$

Here we have picked one of the outgoing massless particles moving in the $x^{1}$ direction and set the scattering point as $\left(t_{0}, t_{0}, 0, y_{0}\right)$ for simplicity. 
The retarded potential is decomposed into the direct and tail parts, $\chi_{a b}^{\mathrm{sw}}=\chi_{a b}^{\mathrm{direct}}+\chi_{a b}^{\mathrm{tail}}$,

$$
\begin{aligned}
& \chi_{a b}^{\text {direct }}=4 G E \frac{y y_{0}}{\Delta L^{2}} n_{a} n_{b}\left[\theta(u-\Delta) \int_{\frac{(t-\Delta)^{2}-\left(x^{1}\right)^{2}-\rho^{2}}{2\left(t-\Delta-x^{1}\right)}}^{\frac{t^{2}-\left(x^{1}\right)^{2}-\rho^{2}}{\sqrt{\left(x^{1}-x^{\prime 1}\right)^{2}+\rho^{2}}}} d x^{\prime 1}\right. \\
& \left.+(\theta(u)-\theta(u-\Delta)) \int_{t_{0}}^{\frac{t^{2}-\left(x^{1}\right)^{2}-\rho^{2}}{2\left(t-x^{1}\right)}} \frac{1}{\sqrt{\left(x^{1}-x^{\prime 1}\right)^{2}+\rho^{2}}} d x^{\prime 1}+(\rho \leftrightarrow \tilde{\rho}, u \leftrightarrow \tilde{u})\right], \\
& \chi_{a b}^{\text {tail }}=-\frac{4 G E}{\Delta L^{2}} n_{a} n_{b}\left[\theta(u) \int_{t_{0}}^{\frac{t^{2}-\left(x^{1}\right)^{2}-\rho^{2}}{2\left(t-x^{1}\right)}}\left(t-x^{\prime 1}-r\right) d x^{\prime 1}-\theta(u-\Delta) \int_{t_{0}}^{\frac{(t-\Delta)^{2}-\left(x^{1}\right)^{2}-\rho^{2}}{2\left(t-\Delta-x^{1}\right)}}\left(t-\Delta-x^{\prime 1}-r\right) d x^{\prime 1}-(\rho \leftrightarrow \tilde{\rho}, r \leftrightarrow \tilde{r}, u \leftrightarrow \tilde{u})\right],
\end{aligned}
$$

where

$$
\begin{array}{cc}
u=t-t_{0}-\sqrt{\left(x^{1}-t_{0}\right)^{2}+\rho^{2}}, \quad \tilde{u}=t-t_{0}-\sqrt{\left(x^{1}-t_{0}\right)^{2}+\tilde{\rho}^{2},} \\
\rho^{2}=\left(x^{2}\right)^{2}+\left(y-y_{0}\right)^{2}, \quad \tilde{\rho}^{2}=\left(x^{2}\right)^{2}+\left(y+y_{0}\right)^{2} .
\end{array}
$$

The shock-wave limit should be taken carefully by first performing the integrations, then taking $t_{0} \rightarrow-\infty$ and finally the limit $\Delta \rightarrow 0$. As a result, we obtain

$$
\begin{gathered}
\chi_{a b}^{\mathrm{direct}} \rightarrow 4 G E \frac{y y_{0}}{L^{2}} n_{a} n_{b}\left[\frac{2}{t-x^{1}} \theta\left(t-x^{1}\right)-\ln \left(\frac{\rho^{2} \tilde{\rho}^{2}}{4 t_{0}^{2}\left(t-x^{1}\right)^{2}}\right) \delta\left(t-x^{1}\right)\right], \\
\chi_{a b}^{\text {tail }} \rightarrow-4 G E \frac{y y_{0}}{L^{2}} n_{a} n_{b}\left[\frac{2}{t-x^{1}} \theta\left(t-x^{1}\right)+\left(2-\ln \left(\frac{\rho^{2} \tilde{\rho}^{2}}{4 t_{0}^{2}\left(t-x^{1}\right)^{2}}\right)-\frac{\rho^{2}+\tilde{\rho}^{2}}{4 y y_{0}} \ln \left(\frac{\tilde{\rho}^{2}}{\rho^{2}}\right)\right) \delta\left(t-x^{1}\right)\right] .
\end{gathered}
$$

Adding together (82) and (83), we obtain

$\lim _{\substack{t_{0 \rightarrow-\infty} \rightarrow \infty \\ \Delta \rightarrow 0}} \chi_{a b}^{\mathrm{sw}}=\frac{8 G E}{L^{2}} n_{a} n_{b}\left[-y y_{0}+\frac{\rho^{2}+\tilde{\rho}^{2}}{8} \ln \left(\frac{\tilde{\rho}^{2}}{\rho^{2}}\right)\right] \delta\left(t-x^{1}\right)$.

This is precisely the AdS shock-wave geometry obtained in $[38,40]$ for $y_{0}=L$. One can check that the AdS shockwave geometry $g_{\mu \nu}=\bar{g}_{\mu \nu}+\delta^{a}{ }_{\mu} \delta^{b}{ }_{\nu} \frac{L^{2}}{y^{2}} \chi_{a b}^{\mathrm{sw}}$ is in fact a solution to the full nonlinear Einstein equation with a source given by a massless particle traveling at the speed of light forever. Thus we have provided an alternative derivation of the AdS shock wave by taking a limit of the retarded potential. We note that it is crucial in our derivation of the shock-wave geometry to take into account the tail term. Otherwise we will not get the correct result. The vacuum expectation value of boundary energy-momentum tensor corresponding to (84) is

$$
\left\langle T_{a b}^{\mathrm{B}}\right\rangle=n_{a} n_{b} \frac{2 E y_{0}^{3}}{\pi\left(\left(x^{2}\right)^{2}+y_{0}^{2}\right)^{2}} \delta\left(t-x^{1}\right),
$$

and this is localized on the light cone $[41,42]$.

\section{E. Velocity memory of AdS shock wave}

It is known that passing through the shock wave causes a jump in the advanced time coordinate and a refraction of the geodesic [43-46]. It is instructive to compare the memory effect of shock wave in AdS spacetime and flat spacetime. In flat space, the shock wave induces a permanent displacement in the relative velocity of two nearby timelike geodesics $[7,34,47,48]$. We will show now that the AdS shock wave induces much richer features in the velocity memory: the relative velocity kick (98) in AdS has a kink $u \theta(u)$, a jump $\theta(u)$ and a pulse $\delta(u)$ in its $v$ component; and a kink and a jump in the $u, x^{2}$ and $y$ components.

To start with, let us review the analysis of [46] for geodesic motion in the AdS shock-wave background. The shock-wave metric is written using a five-dimensional formalism as

$d s^{2}=H\left(Z_{2}, Z_{3}, Z_{4}\right) \delta(U) d U^{2}-2 d U d V+d Z_{2}^{2}+d Z_{3}^{2}-d Z_{4}^{2}$,

where $U=\left(Z_{0}+Z_{1}\right) / \sqrt{2}, \quad V=\left(Z_{0}-Z_{1}\right) / \sqrt{2}$ and the five-dimensional coordinates are subject to a constraint, 


$$
-2 U V+Z_{2}^{2}+Z_{3}^{2}-Z_{4}^{2}=-L^{2} .
$$

In this description, the shock wave is localized at $U=0$. In the following, we assume that $H$ is a function of $Z_{4}$ only $H=H\left(Z_{4}\right)$ so that it corresponds to the Hotta-Tanaka AdS shock wave as [40]

$$
H\left(Z_{4}\right)=\frac{4 \sqrt{2} G E}{L}\left[-2 L+Z_{4} \log \left(\frac{L+Z_{4}}{L-Z_{4}}\right)\right] .
$$

The most general solution to the geodesic equation in the AdS shock-wave background (86) is given by Eq. (39) of [46] with $e=-1$, where it was also pointed out that one can always achieve the vanishing velocities in front of the shock wave, $\dot{Z}_{p}^{0}=d Z_{p} / d \tau\left(U=0^{-}\right)=0, p=2,3,4$, by utilizing the symmetry of the shock-wave background. Thus without loss of generality, one can reduce the solution to the form [Eq. (37) of [46]]

$$
\begin{aligned}
U & =L \dot{U}^{0} \sin \left(\frac{\tau}{L}\right), \\
Z_{p}(U) & =Z_{p}^{0} \sqrt{1-\left(L \dot{U}^{0}\right)^{-2} U^{2}}+A_{p} U \theta(U), \\
V(U) & =\frac{1}{2}\left(\dot{U}^{0}\right)^{-2} U+B \theta(U) \sqrt{1-\left(L \dot{U}^{0}\right)^{-2} U^{2}}+C U \theta(U),
\end{aligned}
$$

where $\tau$ is the proper time of a timelike geodesic and

$$
\begin{aligned}
A_{4} & =\frac{1}{2}\left(-\partial_{4} H(0)+\frac{1}{L^{2}} Z_{4}^{0} G(0)\right) \\
A_{i} & =\frac{1}{2 L^{2}} Z_{i}^{0} G(0), \quad i=2,3 \\
B & =\frac{1}{2} H(0), \\
C & =\frac{1}{8}\left(-\left(\partial_{4} H(0)\right)^{2}-\frac{1}{L^{2}} H(0)^{2}+\left(Z_{4}^{0} \partial_{4} H(0)\right)^{2}\right), \\
H(0) & :=H\left(Z_{4}^{0}\right), \quad G(0):=Z_{4}^{0} \partial_{4} H(0)-H(0) .
\end{aligned}
$$

The solution is specified by the constants $\dot{U}^{0}:=$ $d U / d \tau\left(U=0^{-}\right)$and $Z_{p}^{0}:=Z_{p}\left(U=0^{-}\right)$. We are interested in the geodesic deviation of two nearby timelike geodesics, $x_{A}(\tau)$ and $x_{B}(\tau)$. Before crossing the shock wave at $U=0$, the relative velocity of the two geodesics is generally nonzero and it would not be possible to achieve $\dot{Z}_{p}^{0}=0$ for both of the geodesics by utilizing the symmetry transformations of the coordinates. However, if the two geodesics are parallel to each other and are at the same velocity (the relative velocity is zero) initially, then it is possible to achieve $\dot{Z}_{p}^{0}=0$ for both $x_{A}(\tau)$ and $x_{B}(\tau)$ by using the symmetry transformations. In what follows we will restrict ourselves to this case since it is a very natural situation to have a pair of test particles which are initially at rest at the time the shock wave arrives, and to consider the relative velocity kick induced by the shock wave. As we will see later, in this case the geodesic deviation in the Poincaré coordinates can be characterized by $\xi_{0}$, the relative separation in the $x^{2}$ direction at $U=0_{-}$.

Let us express the above solution (89) in the Poincare coordinates $\left(t, x^{1}, x^{2}, y\right)$ by using the coordinate transformation:

$Z_{0}=\frac{L}{y} t, \quad Z_{1}=-\frac{L}{y} x^{1}$,

$Z_{2}=\frac{L}{y} x^{2}, \quad Z_{3}=\frac{L}{2 y y_{s}}\left(y^{2}+\left(x^{1}\right)^{2}+\left(x^{2}\right)^{2}-t^{2}-y_{s}^{2}\right)$,

$Z_{4}=\frac{L}{2 y y_{s}}\left(y^{2}+\left(x^{1}\right)^{2}+\left(x^{2}\right)^{2}-t^{2}+y_{s}^{2}\right)$,

where $y_{s}$ denotes the value of the $y$ coordinate at the source massless particle. The shock-wave metric (86) can then be written as

$$
d s^{2}=\frac{L^{2}}{y^{2}}\left(\frac{y}{L} H\left(x^{2}, y\right) \delta(u) d u^{2}-2 d u d v+\left(d x^{2}\right)^{2}+d y^{2}\right),
$$

where $u=\left(t-x^{1}\right) / \sqrt{2}, v=\left(t+x^{1}\right) / \sqrt{2}$ and

$$
\begin{aligned}
H\left(x^{2}, y\right)= & 4 \sqrt{2} G E\left[-2+\frac{\left(x^{2}\right)^{2}+y^{2}+y_{s}^{2}}{2 y y_{s}}\right. \\
& \left.\times \log \left(\frac{\left(x^{2}\right)^{2}+\left(y+y_{s}\right)^{2}}{\left(x^{2}\right)^{2}+\left(y-y_{s}\right)^{2}}\right)\right] .
\end{aligned}
$$

Here $t=x^{1}$ has been imposed by $\delta(u)$. Using (91), the solution (89) can be written in terms of $\left(u, v, x^{2}, y\right)$ as

$$
\begin{aligned}
y(U) & =\frac{y_{s} L}{Z_{4}-Z_{3}}=\frac{y_{s} L}{y_{s} \sqrt{1-2 U^{2} L^{-2}}+\left(A_{4}-A_{3}\right) U \theta(U)}, \\
u(U) & =\frac{1}{\sqrt{2}} \frac{Z_{0}+Z_{1}}{Z_{4}-Z_{3}}=\frac{y}{L} U, \\
v(U) & =\frac{1}{\sqrt{2}} \frac{Z_{0}-Z_{1}}{Z_{4}-Z_{3}}=\frac{y}{L}\left[U+\left(B \sqrt{1-2 U^{2} L^{-2}}+C U\right) \theta(U)\right], \\
x^{2}(U) & =\frac{y_{s} Z_{2}}{Z_{4}-Z_{3}}=\frac{y}{L}\left(Z_{2}^{0} \sqrt{1-2 U^{2} L^{-2}}+A_{2} U \theta(U)\right),
\end{aligned}
$$

where we have set $\dot{U}^{0}=1 / \sqrt{2}$ for simplicity. We immediately find that $y\left(U=0^{-}\right)=L$ and then it follows that $x_{0}^{2}:=x^{2}\left(U=0^{-}\right)=Z_{2}^{0}, Z_{3}^{0}=\left(\left(x_{0}^{2}\right)^{2}+L^{2}-y_{s}^{2}\right) /\left(2 y_{s}\right)$ and $Z_{4}^{0}=\left(\left(x_{0}^{2}\right)^{2}+L^{2}+y_{s}^{2}\right) /\left(2 y_{s}\right)$. Hence the solution (94) is specified entirely in terms of $x_{0}^{2}$.

Let us now consider two nearby timelike geodesics denoted by $x_{A}^{\mu}(\tau)$ and $x_{B}^{\mu}(\tau)$ and the geodesic deviation between them. As mentioned in the above, we consider the 
case in which $x_{A}^{\mu}(\tau)$ and $x_{B}^{\mu}(\tau)$ are parallel with the same velocity. Then, let $x_{A}^{\mu}(\tau)$ be specified by (94) with $\left(x_{A}^{2}\right)_{0}=$ $x_{0}^{2}$ and $x_{B}^{\mu}(\tau)$ be that specified by $\left(x_{B}^{2}\right)_{0}=x_{0}^{2}+\xi_{0} \cdot x_{A}(\tau)$ passes the AdS shock wave at $\tau=0, x^{2}=x_{0}^{2}$ while $x_{A}(\tau)$ passes it at $\tau=0, x^{2}=x_{0}^{2}+\xi_{0}$. The geodesic deviation is written as

$$
D^{\mu}=x_{B}^{\mu}(\tau)-x_{A}^{\mu}(\tau)
$$

with

$$
D^{\mu}(U<0)=\xi_{0} \delta_{2}{ }_{2}, \quad \frac{d D^{\mu}}{d U}(U<0)=0 .
$$

At the lowest order of $\xi_{0}$, we obtain

$$
\begin{aligned}
D^{u}= & \frac{D^{y}}{L} U, \\
D^{y}= & -\frac{y_{s} L \partial_{x_{0}^{2}}\left(A_{4}-A_{3}\right) \xi_{0} U \theta(U)}{\left(y_{s} \sqrt{1-2 U^{2} L^{-2}}+\left(A_{4}-A_{3}\right) U \theta(U)\right)^{2}}, \\
D^{v}= & \frac{D^{y}}{L}\left[U+\left(B \sqrt{1-2 U^{2} L^{-2}}+C U\right) \theta(U)\right] \\
& +\frac{y \xi_{0}}{L} \partial_{x_{0}^{2}}\left(B \sqrt{1-2 U^{2} L^{-2}}+C U\right) \theta(U), \\
D^{2}= & \frac{D^{y}}{L}\left(x_{0}^{2} \sqrt{1-2 U^{2} L^{-2}}+A_{2} U \theta(U)\right) \\
& +\frac{y}{L} \xi_{0}\left(\sqrt{1-2 U^{2} L^{-2}}+\partial_{x_{0}^{2}} A_{2} U \theta(U)\right) .
\end{aligned}
$$

The relative velocity kick can be written as

$$
\Delta v^{\mu}:=\frac{d D^{\mu}}{d U}(U>0)-\frac{d D^{\mu}}{d U}(U<0) .
$$

Using the same terminology as in [47], we conclude that $\Delta v^{\mu}$ receives a kink $U \theta(U)$, a jump $\theta(U)$ and a pulse $\delta(U)$ in the $v$-component, and a kink and a jump in the $\left(u, x^{2}, y\right)$ directions.

We remark that it is possible to introduce FNC to investigate the geodesic deviation in the AdS shock-wave background. In that case it is expected from the flat space results $[34]^{5}$ that the geodesic deviation (97) in the FNC is nonzero only in the transverse $x_{F}^{2}$ and $y_{F}$ directions and so

$$
\begin{aligned}
& { }^{5} \text { The flat space result, } \\
& D^{u}=D^{y}=0, \\
& D^{v}=-\xi_{0}\left(\frac{4 \sqrt{2} G E}{x_{0}^{2}} \theta(u)+\frac{32(G E)^{2}}{\left(x_{0}^{2}\right)^{3}} u \theta(u)\right), \\
& D^{2}=\xi_{0}\left(1+\frac{4 \sqrt{2} G E}{\left(x_{0}^{2}\right)^{2}} u \theta(u)\right),
\end{aligned}
$$

can be obtained by taking $y \rightarrow L, y_{s} \rightarrow L, L \rightarrow \infty$ in (97). is the relative velocity kick. We also note that in the FNC the geodesic derivation will not be simply multiplied by the overall scale factor $y / L$, and so it can be inferred from the fourth equations of (94) and (97) that we will have a permanent displacement in the relative velocity kick in the $x^{2}$-direction.

\section{CONCLUSION AND DISCUSSIONS}

We have investigated the retarded potential and memory effect for the particle scattering source in AdS space. Apart from the tail term, the retarded propagator receives contribution from the reflected gravitational waves. We evaluated the retarded potential for a particle scattering source and found that the retarded potential contains two kinds of step functions $\theta(u)$ and $\theta(\tilde{u})$ corresponding to the two types of the gravitational waves, one came directly from the source and the other experienced a reflection. As a consequence, the retarded potential is nonzero only in a finite domain of the spacetime as shown in Fig. 2. Once the two contributions become active, they cancel each other out giving a vanishing retarded potential and the spacetime goes back to the original vacuum AdS space. This is a somewhat surprising result.

We have solved the geodesic deviation equation in the perturbed AdS space by making use of Fermi normal coordinates (FNC) and the tensor $\Omega^{\mu}{ }_{\nu}$. We find that in the FNC, the geodesic perturbation vector and the displacement memory depend linearly and locally on the retarded potential exactly the same way as in the flat space (1). This is a nice result of this work.

Even though it may be expected from the behavior of the retarded potential, we made it clear that there will be no memory for the gravitational wave detector which passes through the region of nonzero retarded potential. On the other hand, for a detector which stays in the region of nonzero retarded potential, the direct and tail contributions together are shown to approach to some constant value at the late time, while the perturbed metric still satisfies the vacuum Einstein equation. This corresponds precisely to a nonvanishing memory induced by the gravitational radiation.

It is known that the Fefferman-Graham expansion of the metric near the AdS boundary $(y=0)$ of an asymptotically AdS space begins with the $\mathcal{O}\left(y^{3}\right)$ term and it does not have terms linear or quadratic in $y$. Actually we saw that the asymptotic expansion of the retarded potential for a particle scattering source also begins with the $\mathcal{O}\left(y^{3}\right)$ term as the $\mathcal{O}\left(y^{0}\right)$ and $\mathcal{O}\left(y^{2}\right)$ terms cancels out between the contributions of the gravitational wave with its reflection. It is interesting to understand better if and how the asymptotic form of the memory is related to the asymptotic symmetries of AdS space.

We have considered the memory effect of a shock wave in AdS. We find for a pair of particles initially at rest relative to each other, the passage of the shock wave will 
induce a velocity kick in the relative velocity. Unlike in the flat case where the velocity kick is in the form of a jump $\theta(u)$ and a pulse $\delta(u)$, there is also a kink $u \theta(u)$ contribution in the AdS case.

In flat spacetime, the gravitational memory effect from the localized particle source is characterized [7] by a discontinuity in the retarded potential and a first order derivative of the delta function in the Riemann tensor. In the present AdS case, the retarded potential in AdS space gets additional complications compared to that in the flat space: there is the multiplication of the warp factor $y y_{0} / L^{2}$ and there is also an additional contribution from the reflected gravitational waves proportional to $\theta(\tilde{u})$.

In this work, we have demonstrated that the use of the Fermi normal coordinates allows us to disentangle in the geodesic deviation the background curvature contribution from the gravitational wave contribution, and extract the gravitational memory of interest. For general curved spacetime, our analysis suggests that the use of a certain adapted coordinate system could be very helpful in allowing one to dissect the geodesic deviation of test particles and extract the relevant memory due to gravitational radiation. It is interesting to understand what properties are needed for the right local coordinate system. For the de Sitter space, due to the background expansion, we find that the use of conformal Fermi coordinates (CNC) seems to be the right choice. This is an interesting direction for further exploration [28].

\section{ACKNOWLEDGMENTS}

We would like to thank Calros Cardona, Dimitrios Giataganas, Wu-Zhong Guo, Yuta Hamada, Hiroyuki Kitamoto, Toshifumi Noumi, Sang-Jin Sin, and Gary Shiu for valuable discussion and comments. This work is supported in part by the National Center for Theoretical Sciences (NCTS) and Grant No. 107-2119-M-007-014-MY3 from the Ministry of Science and Technology of Taiwan.

\section{APPENDIX A: GEODESIC EQUATION IN AdS SPACE}

Geodesic equations for a point particle in the vacuum AdS space (5) are

$\ddot{t}-\frac{2}{y} \dot{t} \dot{y}=0, \quad \ddot{x}^{i}-\frac{2}{y} \dot{x}^{i} \dot{y}=0, \quad \ddot{y}-\frac{1}{y}\left(\dot{t}^{2}+\dot{y}^{2}-\delta_{i j} \dot{x}^{i} \dot{x}^{j}\right)=0$,

where the dot denotes a derivative with respect to the proper time for massive particles or the affine parameter for massless particles. There is a constraint equation

$$
\frac{L^{2}}{y^{2}}\left(-\dot{t}^{2}+\delta_{i j} \dot{x}^{i} \dot{x}^{j}+\dot{y}^{2}\right)= \begin{cases}-1 & (\text { massive }) \\ 0 & (\text { massless }) .\end{cases}
$$

Conserved quantities $P_{a}$ in the geodesic motion are given by

$P_{0} / m=-\bar{g}_{00} u^{0}=\frac{L^{2}}{y^{2}} \dot{t}, \quad P_{i} / m=\bar{g}_{i j} u^{j}=\frac{L^{2}}{y^{2}} \delta_{i j} \dot{x}^{j}$.

The energy of particle measured by a timelike observer whose 4-velocity is $t^{\mu}$ is

$$
E=-P_{\mu} t^{\mu}
$$

For a timelike Killing vector $t^{\mu}$ in AdS space, $t^{\mu}=$ $(-1,0,0,0), E=P_{0}$. Using (A2) and (A3), (A1) becomes

$$
\begin{aligned}
& \ddot{t}=\frac{2 y}{L^{2}} P_{0} \dot{y}, \quad \ddot{x}^{i}=\frac{2 y}{L^{2}} P^{i} \dot{y}, \\
& \ddot{y}= \begin{cases}\frac{2 y^{3}}{L^{4}}\left(\frac{P_{0}^{2}}{m^{2}}-\frac{P_{i}^{2}}{m^{2}}-\frac{L^{2}}{2 y^{2}}\right) & \text { (massive) } \\
\frac{2 y^{3}}{L^{4}}\left(P_{0}^{2}-P_{i}^{2}\right) & \text { (massless), }\end{cases}
\end{aligned}
$$

where $P^{i}=\delta^{i j} P_{j}, P_{i}^{2}=\delta^{i j} P_{i} P_{j}$.

For a massive particle, from (A3) and (A2), we have

$$
\begin{aligned}
& \dot{x}^{i}=\frac{y^{2}}{L^{2}} \frac{P_{0}}{m} \frac{d x^{i}}{d t}=\frac{y^{2}}{L^{2}} \frac{P^{i}}{m} \\
& \dot{y}^{2}=\frac{y^{4}}{L^{4}} \frac{P_{0}^{2}}{m^{2}}\left(\frac{d y}{d t}\right)^{2}=\frac{y^{4}}{L^{4}}\left(\frac{P_{0}^{2}-P_{i}^{2}}{m^{2}}\right)-\frac{y^{2}}{L^{2}} .
\end{aligned}
$$

It follows that

$x^{i}=\frac{P^{i}}{P_{0}} t+C^{i}, \quad y=\sqrt{\frac{m^{2} L^{2}+P_{0}^{-2}\left(P_{0}^{2}-P_{i}^{2}\right)^{2}\left(t-C_{3}\right)^{2}}{P_{0}^{2}-P_{i}^{2}}}$,

where $C_{i}$ and $C_{3}$ are constants and $C_{3}$ determines the time at which $y$ takes minimum value. Taking $m \rightarrow 0$ in (A7) gives a solution to the geodesic equation of a massless particle. The energy of the massless particle is written as $E=L^{2} y^{-2} \dot{t}$.

\section{APPENDIX B: LINEARIZED RIEMANN TENSOR}

The Christoffel symbols for the background AdS metric (5) are

$$
\bar{\Gamma}_{\mu \nu}^{\rho}=-\frac{1}{y}\left(\delta_{\mu}^{y} \delta_{\nu}^{\rho}+\delta_{\nu}^{y} \delta_{\mu}^{\rho}-\delta_{y}^{\rho} \eta_{\mu \nu}\right) .
$$

The linearized Riemann tensor is given by

$$
\begin{aligned}
R_{\alpha \beta \gamma}^{\mu(1)}= & \frac{1}{2}\left(\bar{\nabla}_{\beta} \bar{\nabla}_{\alpha} \gamma_{\gamma}^{\mu}-\bar{\nabla}_{\gamma} \bar{\nabla}_{\alpha} \gamma_{\beta}^{\mu}+\bar{\nabla}_{\gamma} \bar{\nabla}^{\mu} \gamma_{\alpha \beta}-\bar{\nabla}_{\beta} \bar{\nabla}^{\mu} \gamma_{\alpha \gamma}\right. \\
& \left.+\bar{R}_{\rho \beta \gamma}^{\mu} \gamma_{\alpha}{ }_{\alpha}-\bar{R}_{\alpha \beta \gamma}^{\rho} \gamma_{\rho}^{\mu}\right) .
\end{aligned}
$$


Substituting (5) and (B1) into (B2), we get

$$
\begin{aligned}
R_{\alpha \beta \gamma}^{\mu(1)}= & A_{\alpha \beta \gamma}^{\mu}-A_{\alpha \gamma \beta}^{\mu}-\bar{g}^{\mu \rho} \bar{g}_{\alpha \sigma}\left(A^{\sigma}{ }_{\rho \beta \gamma}-A^{\sigma}{ }_{\rho \gamma \beta}\right) \\
& -\frac{1}{2 y^{2}}\left(\delta_{\beta}^{\mu} \eta_{\rho \gamma} \gamma_{\alpha}^{\rho}-\delta_{\gamma}^{\mu} \eta_{\rho \beta} \gamma_{\alpha}^{\rho}-\delta_{\beta}^{\rho} \eta_{\alpha \gamma} \gamma_{\rho}^{\mu}+\delta_{\gamma}^{\rho} \eta_{\alpha \beta} \gamma_{\rho}^{\mu}\right),
\end{aligned}
$$

where

$$
\begin{aligned}
A_{\alpha \beta \gamma}^{\mu}= & \frac{1}{2}\left[\partial_{\beta} \partial_{\alpha} \gamma_{\gamma}^{\mu}-\frac{1}{y}\left(\delta_{\alpha}^{\mu} \partial_{\beta} \gamma^{y}{ }_{\gamma}-\frac{y^{2}}{L^{2}} \delta_{y}^{\mu} \partial_{\beta} \gamma_{\alpha \gamma}-\delta_{\gamma}^{y} \partial_{\beta} \gamma^{\mu}{ }_{\alpha}\right.\right. \\
& +\eta_{\gamma \alpha} \partial_{\beta} \gamma^{\mu}{ }_{y}-\delta_{\alpha}^{y} \partial_{\beta} \gamma^{\mu}{ }_{\gamma}+\eta_{\beta \alpha} \partial_{y} \gamma^{\mu}{ }_{\gamma}+\delta_{\beta}^{\mu} \partial_{\alpha} \gamma^{y}{ }_{\gamma} \\
& \left.-\delta_{y}^{\mu} \eta_{\beta \rho} \partial_{\alpha} \gamma^{\rho}{ }_{\gamma}\right)+\frac{1}{y^{2}}\left(\delta_{\beta}^{y} \delta_{\alpha}^{\mu} \gamma_{\gamma}^{y}+\delta_{\alpha}^{y} \delta_{\gamma}^{y} \gamma^{\mu}{ }_{\beta}\right. \\
& -\frac{y^{2}}{L^{2}} \delta_{\beta}^{\mu} \gamma_{\alpha \gamma}-\delta_{\beta}^{\mu} \delta_{\gamma}^{y} \gamma_{\alpha}^{y}+\delta_{\beta}^{\mu} \eta_{\alpha \gamma} \gamma^{y}{ }_{y} \\
& \left.\left.+\frac{y^{2}}{L^{2}} \delta_{y}^{\mu} \delta_{\beta}^{y} \gamma_{\alpha \gamma}-\delta_{\gamma}^{y} \eta_{\beta \alpha} \gamma_{y}{ }_{y}\right)\right] .
\end{aligned}
$$

\section{APPENDIX C: GEODESIC AND PARALLEL TRANSPORT EQUATIONS IN PERTURBED AdS SPACE}

Here we consider the geodesic and geodesic deviation equations in AdS space with perturbations. Our analysis is restricted to the first order of the metric perturbations.

\section{Perturbed geodesic and parallel transport equations}

A formalism to construct and solve the perturbed geodesic equation in curved spaces is given in [49,50]. One first decomposes the geodesic into the background trajectory and perturbation about it:

$$
x^{\mu}(\tau)=\bar{x}^{\mu}(\tau)+\delta x^{\mu}(\tau),
$$

where $\bar{x}^{\mu}$ solves the background geodesic equation,

$$
\frac{d^{2} \bar{x}^{\mu}}{d \tau^{2}}+\bar{\Gamma}_{\alpha \beta}^{\mu} \frac{d \bar{x}^{\alpha}}{d \tau} \frac{d \bar{x}^{\beta}}{d \tau}=0
$$

with $\bar{\Gamma}_{\alpha \beta}^{\mu}$ the Christoffel symbols consisting of the background metric, and $\delta x^{\mu}$ is $\mathcal{O}\left(\gamma_{\mu \nu}\right)$ quantity. ${ }^{6}$ Substituting (C1) into the geodesic equation,

$$
\frac{d^{2} x^{\mu}}{d \tau^{2}}+\Gamma_{\alpha \beta}^{\mu}(x) \frac{d x^{\alpha}}{d \tau} \frac{d x^{\beta}}{d \tau}=0,
$$

\footnotetext{
${ }^{6}$ For notation simplicity, in the main body (Sec. III) we are using $x^{\mu}(\tau)$ as the background geodesic $\bar{x}^{\mu}(\tau)$ as long as there is no confusion.
}

one obtains at the first order of perturbation that

$$
\left(\delta^{\mu}{ }_{\beta} \frac{d^{2}}{d \tau^{2}}+A^{\mu}{ }_{\beta} \frac{d}{d \tau}+B_{\beta}^{\mu}\right) \delta x^{\beta}=f^{\mu}
$$

where

$$
\begin{aligned}
A_{\beta}^{\mu} & =2 \bar{\Gamma}_{\alpha \beta}^{\mu} \frac{d \bar{x}^{\alpha}}{d \tau}, \quad B_{\beta}^{\mu}=\partial_{\beta}\left(\bar{\Gamma}_{\alpha \rho}^{\mu}\right) \frac{d \bar{x}^{\alpha}}{d \tau} \frac{d \bar{x}^{\rho}}{d \tau}, \\
f^{\mu} & =-\delta \Gamma_{\alpha \beta}^{\mu} \frac{d \bar{x}^{\alpha}}{d \tau} \frac{d \bar{x}^{\beta}}{d \tau} .
\end{aligned}
$$

In our case of the Poincaré coordinates in AdS space, $f^{\mu}$ is given by

$$
f^{\mu}=-\left(\partial_{\alpha} h_{\beta}^{\mu}-\frac{1}{2} \partial^{\mu} h_{\alpha \beta}+\frac{1}{y} \delta_{y}^{\mu} h_{\alpha \beta}-\frac{1}{y} \eta_{\alpha \beta} h^{y \mu}\right) \frac{d \bar{x}^{\alpha}}{d \tau} \frac{d \bar{x}^{\beta}}{d \tau}
$$

where $h_{\mu \nu}:=y^{2} L^{-2} \gamma_{\mu \nu}$.

Corresponding to (C1), we also decompose the tetrads into its background and perturbation pieces

$$
\left(e_{\alpha}\right)^{\mu}(\tau)=\left.\frac{\partial x^{\mu}}{\partial x_{F}^{\alpha}}\right|_{\gamma(\tau)}=\bar{e}_{\alpha}^{\mu}(\tau)+\delta e_{\alpha}^{\mu}(\tau),
$$

where $\bar{e}_{\alpha}^{\mu}$ solves the background constraint equation, $\bar{g}_{\mu \nu}(\bar{x}) \bar{e}_{\alpha}^{\mu} \bar{e}_{\beta}^{\nu}=\eta_{\alpha \beta}$. Note that $\bar{e}_{0}^{\mu}=d \bar{x}^{\mu} / d \tau$ and $\delta e_{0}^{\mu}=d \delta x^{\mu} /$ $d \tau$. Substituting (C1) and (C7) into the constraint equation, $g_{\mu \nu}(x)\left(e_{\alpha}\right)^{\mu}\left(e_{\beta}\right)^{\nu}=\eta_{\alpha \beta}$, and the parallel transport equation, $d\left(e_{\alpha}\right)^{\mu} / d \tau+\Gamma_{\rho \sigma}^{\mu}(x)\left(e_{0}\right)^{\rho}\left(e_{\alpha}\right)^{\sigma}=0$, we obtain

$$
2 \bar{g}_{\mu \nu} \bar{e}_{\alpha}^{\mu} \delta e_{\beta}^{\nu}+\left(\gamma_{\mu \nu}+\partial_{\rho}\left(\bar{g}_{\mu \nu}\right) \delta x^{\rho}\right) \bar{e}_{\alpha}^{\mu} \bar{e}_{\beta}^{\nu}=0,
$$

$$
\frac{d}{d \tau} \delta e_{\alpha}^{\mu}+\bar{\Gamma}_{\rho \sigma}^{\mu}\left(\delta e_{0}^{\rho} \bar{e}_{\alpha}^{\sigma}+\bar{e}_{0}^{\rho} \delta e_{\alpha}^{\sigma}\right)\left(\delta \Gamma_{\alpha \beta}^{\mu}+\partial_{\beta}\left(\bar{\Gamma}_{\rho \sigma}^{\mu}\right) \delta x^{\beta}\right) \bar{e}_{0}^{\rho} \bar{e}_{\alpha}^{\sigma}=0 .
$$

\section{Solution to the perturbed geodesic equation}

A general solution to $(\mathrm{C} 4)$ can be represented by using the parallel propagator and the Jacobi propagator [50] as follows:

$$
\begin{aligned}
& \left(\begin{array}{c}
\frac{1}{L} P\left(\tau_{i}, \tau\right) \delta x(\tau) \\
\frac{d}{d \tau}\left(P\left(\tau_{i}, \tau\right) \delta x(\tau)\right)
\end{array}\right) \\
& =U\left(\tau, \tau_{i}\right)\left(\begin{array}{c}
\frac{1}{L} \delta x\left(\tau_{i}\right) \\
\left.\frac{d}{d \tau^{\prime}}\left(P\left(\tau_{i}, \tau^{\prime}\right) \delta x\left(\tau^{\prime}\right)\right)\right|_{\tau^{\prime}=\tau_{i}}
\end{array}\right) \\
& \quad+\int_{\tau_{i}}^{\tau} d \bar{\tau} U(\tau, \bar{\tau})\left(\begin{array}{c}
0_{4} \\
P\left(\tau_{i}, \bar{\tau}\right) f(\bar{\tau})
\end{array}\right),
\end{aligned}
$$


where we have adopted the matrix notation, e.g., $P \delta x=$ $P^{\mu}{ }_{\alpha} \delta x^{\alpha}, P f=P^{\mu}{ }_{\alpha} f^{\alpha}$, and $L$ is some length scale which is identified with the AdS radius in our case. The parallel propagator $P\left(\tau_{1}, \tau_{2}\right)$ is given by a $4 \times 4$ matrix,

where $\mathcal{P}$ denotes the path ordering and $A$ is given by $A^{\mu}{ }_{\beta}$ in (C5), and the Jacobi propagator $U\left(\tau_{1}, \tau_{2}\right)$ is given by an $8 \times 8$ matrix,

$P\left(\tau_{1}, \tau_{2}\right)^{\mu}{ }_{\nu}=\mathcal{P} \exp \left(-\frac{1}{2} \int_{\tau_{2}}^{\tau_{1}} d \tau A(\tau)\right)_{\nu}^{\mu}$,

$$
P\left(\tau_{1}, \tau_{2}\right)=P^{-1}\left(\tau_{2}, \tau_{1}\right) \text {, }
$$

$U\left(\tau_{1}, \tau_{2}\right)=\mathcal{P} \exp \left[\frac{1}{L} \int_{\tau_{2}}^{\tau_{1}} d \tau\left(\begin{array}{cc}0 & 1_{4} \\ -P\left(\tau_{1}, \tau\right) \mathcal{R}(\tau) P\left(\tau, \tau_{1}\right) & 0\end{array}\right)\right]$,

where $\mathcal{R}_{\nu}^{\mu}:=\bar{R}_{\alpha \nu \beta}^{\mu} \bar{e}_{0}^{\alpha} \bar{e}_{0}^{\beta}$. Note that the first term on the right-hand side of (C10) vanishes for a set of initial conditions $\delta x^{\mu}\left(\tau_{i}\right)=d \delta x^{\mu} / d \tau\left(\tau_{i}\right)=0$.

For the central geodesic $\gamma(\tau)$ in the AdS space, the parallel and Jacobi propagators are obtained as

$$
\begin{aligned}
& P\left(\tau_{1}, \tau_{2}\right)=\left(\begin{array}{cccc}
\frac{\bar{y}\left(\tau_{1}\right)^{2}}{L^{2}}\left(1-\frac{\bar{z}\left(\tau_{1}\right) \bar{t}\left(\tau_{2}\right)}{\bar{y}\left(\tau_{1}\right) \bar{y}\left(\tau_{2}\right)}\right) & 0 & 0 & \frac{\bar{y}\left(\tau_{1}\right)^{2}}{L^{2}}\left(\frac{\bar{F}\left(\tau_{1}\right)}{\bar{y}\left(\tau_{1}\right)}-\frac{\bar{z}\left(\tau_{2}\right)}{\bar{y}\left(\tau_{2}\right)}\right) \\
0 & \bar{y}\left(\tau_{1}\right) & 0 & 0 \\
0 & 0 & \frac{\bar{y}\left(\tau_{1}\right)}{\bar{y}\left(\tau_{2}\right)} & 0 \\
\frac{\bar{y}\left(\tau_{1}\right)^{2}}{L^{2}}\left(\frac{\bar{t}\left(\tau_{1}\right)}{\bar{y}\left(\tau_{1}\right)}-\frac{\bar{y}\left(\tau_{2}\right)}{\bar{y}\left(\tau_{2}\right)}\right) & 0 & 0 & \frac{\bar{y}\left(\tau_{1}\right)^{2}}{L^{2}}\left(1-\frac{\bar{t}\left(\tau_{1}\right) \bar{f}\left(\tau_{2}\right)}{\bar{y}\left(\tau_{1}\right) \bar{y}\left(\tau_{2}\right)}\right)
\end{array}\right), \\
& U\left(\tau_{1}, \tau_{2}\right)=\left(\begin{array}{cc}
J \cos \left(\frac{\tau_{1}-\tau_{2}}{L}\right) & J \sin \left(\frac{\tau_{1}-\tau_{2}}{L}\right) \\
-J \sin \left(\frac{\tau_{1}-\tau_{2}}{L}\right) & J \cos \left(\frac{\tau_{1}-\tau_{2}}{L}\right)
\end{array}\right)+\left(\begin{array}{cc}
1_{4}-J & \left(1_{4}-J\right) \frac{\tau_{1}-\tau_{2}}{L} \\
0 & 1_{4}-J
\end{array}\right),
\end{aligned}
$$

where

$$
J:=L^{2} P\left(\tau_{1}, \tau\right) \mathcal{R}(\tau) P\left(\tau, \tau_{1}\right)=\left(\begin{array}{cccc}
-\frac{\bar{y}\left(\tau_{1}\right)^{2}}{L^{2}} & 0 & 0 & \frac{\bar{y}\left(\tau_{1}\right) \bar{t}\left(\tau_{1}\right)}{L^{2}} \\
0 & 1 & 0 & 0 \\
0 & 0 & 1 & 0 \\
-\frac{\bar{y}\left(\tau_{1}\right) \bar{t}\left(\tau_{1}\right)}{L^{2}} & 0 & 0 & \frac{\bar{y}\left(\tau_{1}\right)^{2}}{L^{2}}
\end{array}\right)
$$

[1] Ya. B. Zeldovich and A. G. Polnarev, Radiation of gravitational waves by a cluster of superdense stars, Sov. Astron. 18, 17 (1974).

[2] V. B. Braginsky and K. S. Thorne, Gravitational-wave bursts with memory and experimental prospects, Nature (London) 327, 123 (1987).

[3] K. S. Thorne, Gravitational-wave bursts with memory: The Christodoulou effect, Phys. Rev. D 45, 520 (1992).

[4] L. Bieri, P. Chen, and S. T. Yau, The electromagnetic Christodoulou memory effect and its application to neutron star binary mergers, Classical Quantum Gravity 29, 215003 (2012).

[5] L. Bieri and D. Garfinkle, Neutrino radiation showing a christodoulou memory effect in general relativity, Ann. Inst. Henri Poincaré 16, 801 (2015).
[6] L. Bieri and D. Garfinkle, Perturbative and gauge invariant treatment of gravitational wave memory, Phys. Rev. D 89, 084039 (2014).

[7] A. Tolish and R. M. Wald, Retarded fields of null particles and the memory effect, Phys. Rev. D 89, 064008 (2014).

[8] D. Garfinkle, S. Hollands, A. Ishibashi, A. Tolish, and R. M. Wald, The memory effect for particle scattering in even spacetime dimensions, Classical Quantum Gravity 34, 145015 (2017).

[9] A. G. Wiseman and C. M. Will, Christodoulou's nonlinear gravitational wave memory: Evaluation in the quadrupole approximation, Phys. Rev. D 44, R2945 (1991).

[10] M. Favata, The gravitational-wave memory effect, Classical Quantum Gravity 27, 084036 (2010). 
[11] N. Seto, Search for memory and inspiral gravitational waves from supermassive binary black holes with pulsar timing arrays, Mon. Not. R. Astron. Soc. 400, L38 (2009).

[12] R. van Haasteren and Y. Levin, Gravitational-wave memory and pulsar timing arrays, Mon. Not. R. Astron. Soc. 401, 2372 (2010).

[13] P. D. Lasky, E. Thrane, Y. Levin, J. Blackman, and Y. Chen, Detecting Gravitational-Wave Memory with LIGO: Implications of GW150914, Phys. Rev. Lett. 117, 061102 (2016).

[14] H. Bondi, M. G. J. van der Burg, and A. W. K. Metzner, Gravitational waves in general relativity. 7. Waves from axisymmetric isolated systems, Proc. R. Soc. A 269, 21 (1962).

[15] R. K. Sachs, Gravitational waves in general relativity. 8. Waves in asymptotically flat space-times, Proc. R. Soc. A 270, 103 (1962).

[16] A. Strominger and A. Zhiboedov, Gravitational memory, BMS supertranslations and soft theorems, J. High Energy Phys. 01 (2016) 086.

[17] A. Strominger, Lectures on the infrared structure of gravity and gauge theory, arXiv:1703.05448.

[18] L. Bieri, D. Garfinkle, and S. T. Yau, Gravitational wave memory in de Sitter spacetime, Phys. Rev. D 94, 064040 (2016).

[19] Y.Z. Chu, Gravitational wave memory in $\mathrm{dS}_{4+2 n}$ and 4D cosmology, Classical Quantum Gravity 34, 035009 (2017).

[20] Y. Hamada, M. S. Seo, and G. Shiu, Memory in de Sitter space and Bondi-Metzner-Sachs-like supertranslations, Phys. Rev. D 96, 023509 (2017).

[21] A. Kehagias and A. Riotto, BMS in cosmology, J. Cosmol. Astropart. Phys. 05 (2016) 059.

[22] A. Tolish and R. M. Wald, Cosmological memory effect, Phys. Rev. D 94, 044009 (2016).

[23] L. Bieri, D. Garfinkle, and N. Yunes, Gravitational wave memory in $\Lambda \mathrm{CDM}$ cosmology, Classical Quantum Gravity 34, 215002 (2017).

[24] A. Ashtekar, B. Bonga, and A. Kesavan, Asymptotics with a positive cosmological constant: III. The quadrupole formula, Phys. Rev. D 92, 104032 (2015).

[25] A. Ashtekar, B. Bonga, and A. Kesavan, Gravitational Waves from Isolated Systems: Surprising Consequences of a Positive Cosmological Constant, Phys. Rev. Lett. 116, 051101 (2016).

[26] R. K. Mishra, A. Mohd, and R. Sundrum, AdS asymptotic symmetries from CFT mirrors, J. High Energy Phys. 03 (2019) 017.

[27] J. M. Maldacena, The large N limit of superconformal field theories and supergravity, Int. J. Theor. Phys. 38, 1113 (1999); Adv. Theor. Math. Phys. 2, 231 (1998).

[28] C. S. Chu and Y. Koyama (to be published).

[29] H. J. de Vega, J. Ramirez, and N. G. Sanchez, Generation of gravitational waves by generic sources in de Sitter spacetime, Phys. Rev. D 60, 044007 (1999).

[30] U. H. Danielsson, E. Keski-Vakkuri, and M. Kruczenski, Vacua, propagators, and holographic probes in AdS/CFT, J. High Energy Phys. 01 (1999) 002.
[31] F. K. Manasse and C. W. Misner, Fermi normal coordinates and some basic concepts in differential geometry, J. Math. Phys. (N.Y.) 4, 735 (1963).

[32] L. Dai, E. Pajer, and F. Schmidt, Conformal Fermi coordinates, J. Cosmol. Astropart. Phys. 11 (2015) 043.

[33] E. Poisson, A Relativist's Toolkit: The Mathematics of Black-Hole Mechanics (Cambridge University Press, Cambridge, England, 2009).

[34] G. M. Shore, Memory, Penrose limits and the geometry of gravitational shock waves and gyratons, J. High Energy Phys. 12 (2018) 133.

[35] P.C. Aichelburg and R. U. Sexl, On the gravitational field of a massless particle, Gen. Relativ. Gravit. 2, 303 (1971).

[36] R. M. Wald, General Relativity (University of Chicago Press, Chicago, 1984).

[37] S. de Haro, S. N. Solodukhin, and K. Skenderis, Holographic reconstruction of space-time and renormalization in the AdS/CFT correspondence, Commun. Math. Phys. 217, 595 (2001).

[38] M. Hotta and M. Tanaka, Shock wave geometry with nonvanishing cosmological constant, Classical Quantum Gravity 10, 307 (1993).

[39] N. A. Voronov and I. Y. Kobzarev, On the gravitational field of a massless particle, Zh. Eksp. Teor. Fiz. 66, 1179 (1974).

[40] J. Podolsky and J. B. Griffiths, Impulsive gravitational waves generated by null particles in de Sitter and anti-de Sitter backgrounds, Phys. Rev. D 56, 4756 (1997).

[41] G. T. Horowitz and N. Itzhaki, Black holes, shock waves, and causality in the AdS/CFT correspondence, J. High Energy Phys. 02 (1999) 010.

[42] S. S. Gubser, S. S. Pufu, and A. Yarom, Entropy production in collisions of gravitational shock waves and of heavy ions, Phys. Rev. D 78, 066014 (2008).

[43] R. Penrose, Structure of space-time, in Battelle Rencontres, 1967 Lectures in Mathematics and Physics, edited by C. M. DeWitt and J. A. Wheeler (Benjamin, New York, 1968), Sec. 9, pp. 121-235.

[44] T. Dray and G. 't Hooft, The gravitational shock wave of a massless particle, Nucl. Phys. B253, 173 (1985).

[45] K. Sfetsos, On gravitational shock waves in curved spacetimes, Nucl. Phys. B436, 721 (1995).

[46] J. Podolsky and M. Ortaggio, Symmetries and geodesics in (anti-)de Sitter space-times with nonexpanding impulsive waves, Classical Quantum Gravity 18, 2689 (2001).

[47] R. Steinbauer, Geodesics and geodesic deviation for impulsive gravitational waves, J. Math. Phys. (N.Y.) 39, 2201 (1998).

[48] P.-M. Zhang, C. Duval, and P. A. Horvathy, Memory effect for impulsive gravitational waves, Classical Quantum Gravity 35, 065011 (2018).

[49] T. Pyne and M. Birkinshaw, Null geodesics in perturbed space-times, Astrophys. J. 415, 459 (1993).

[50] T. Pyne and S. M. Carroll, Higher order gravitational perturbations of the cosmic microwave background, Phys. Rev. D 53, 2920 (1996). 